OPEN ACCESS

Edited by:

Ela B. Plow,

Cleveland Clinic Foundation, USA

Reviewed by:

Nikhil Sharma

University College London, UK

Patrick Ragert,

Max Planck Institute for Human Cognitive and Brain Sciences Leipzig,

Germany

Kelsey A. Potter-Baker,

Cleveland Clinic Foundation, USA

*Correspondence:

Friedhelm C. Hummel,

Brain Imaging and Neurostimulation

(BINS) Laboratory, Department of Neurology, University Medical Center Hamburg-Eppendorf, Martinistr. 52,

Hamburg 20246, Germany f.humme/@uke.de

${ }^{\dagger}$ Maximilian J. Wessel and Máximo Zimerman have contributed equally to this work

Received: 25 January 2015 Accepted: 23 April 2015 Published: 15 May 2015

Citation:

Wessel MJ, Zimerman M and Hummel FC (2015) Non-invasive brain stimulation: an interventional tool for enhancing behavioral training after stroke.

Front. Hum. Neurosci. 9:265 doi: 10.3389/fnhum.2015.00265

\section{Non-invasive brain stimulation: an interventional tool for enhancing behavioral training after stroke}

\author{
Maximilian J. Wessel ${ }^{1 \dagger}$, Máximo Zimerman ${ }^{1,2+}$ and Friedhelm C. Hummel ${ }^{1,3 *}$ \\ 'Brain Imaging and Neurostimulation (BINS) Laboratory, Department of Neurology, University Medical Center Hamburg- \\ Eppendorf, Hamburg, Germany, ${ }^{2}$ Institute of Cognitive Neurology (INECO), Buenos Aires, Argentina, ${ }^{3}$ Favaloro University, \\ Buenos Aires, Argentina
}

Stroke is the leading cause of disability among adults. Motor deficit is the most common impairment after stroke. Especially, deficits in fine motor skills impair numerous activities of daily life. Re-acquisition of motor skills resulting in improved or more accurate motor performance is paramount to regain function, and is the basis of behavioral motor therapy after stroke. Within the past years, there has been a rapid technological and methodological development in neuroimaging leading to a significant progress in the understanding of the neural substrates that underlie motor skill acquisition and functional recovery in stroke patients. Based on this and the development of novel non-invasive brain stimulation (NIBS) techniques, new adjuvant interventional approaches that augment the response to behavioral training have been proposed. Transcranial direct current, transcranial magnetic, and paired associative (PAS) stimulation are NIBS techniques that can modulate cortical excitability, neuronal plasticity and interact with learning and memory in both healthy individuals and stroke patients. These techniques can enhance the effect of practice and facilitate the retention of tasks that mimic daily life activities. The purpose of the present review is to provide a comprehensive overview of neuroplastic phenomena in the motor system during learning of a motor skill, recovery after brain injury, and of interventional strategies to enhance the beneficial effects of customarily used neurorehabilitation after stroke.

Keywords: stroke, motor learning, non-invasive brain stimulation, motor recovery, TMS, tDCS, neurorehabilitation

\section{Background}

Stroke is a leading cause of serious long-term disability (Kochanek et al., 2012) with growing impact on actual and future health economy. It is estimated that an additional four million people in the US will suffer a stroke by 2030, due to changes in demographic evolution (Heidenreich et al., 2011). This increase in the aging population will result in more demands on health services as stroke in older people often result in more severe functional loss (Baztan et al., 2007).

A large proportion of the focus of stroke research still remains on the acute management of stroke. The development of thrombolytic therapy, determination of an individualized time window to apply thrombolysis (Thomalla et al., 2011), and reduction in early post-stroke complications due to the application of the stroke unit concept, has led to a significant decline of mortality rate after stroke (Langhorne et al., 1993). On the other hand, a substantial proportion of stroke victims are left with moderate to severe disability. Indeed, the resulting motor deficit, especially of the upper extremity, 
has a great impact on activities of daily life. Currently, recovery of hand motor function in a large part of the survivors (55-75\%) is unsatisfying (Nakayama et al., 1994; Jorgensen, 1996; Jorgensen et al., 1999).

As a result, there is a strong need for new innovative strategies to improve stroke rehabilitation. Comprehensive evidence indicates that motor learning mechanisms are operative during spontaneous stroke recovery and interact with rehabilitative training (Krakauer, 2006). As a matter of principle, re-acquisition of skills resulting in improved or more accurate motor performance is paramount for recovery of motor function after a brain lesion. Thus, to a significant degree, the success of neurorehabilitation depends on the amount and effectiveness of rehabilitative training to promote the re-acquisition of motor skill that were lost due to the lesion. In this context, non-invasive brain stimulation (NIBS) techniques have the appeal of being able to specifically and selectively enhance adaptive patterns of activity, suppress maladaptive patterns, and interact directly with the process of motor skill acquisition by sharing synergistic impacts on synaptic plasticity and network reorganization (Bolognini et al., 2009; Reis et al., 2009a; Fritsch et al., 2010).

Here, we review basic principles of motor learning. Further, we focus on modulation of its principles by NIBS and innovative training regimes.

\section{Principles of Motor Learning}

Learning of a motor skill is commonly defined as a process of increased spatial and temporal accuracy of movements with practice (Willingham, 1998). The learning process consists of different temporal components; for illustration, please see Figure 1. In principle, online and offline processes can be segregated. Online learning refers to within-session, and offline learning to in-between session improvement (Robertson et al., 2004a).
During online learning, a first fast learning phase is characterized by rapid improvement usually within in a single training session. Whereas, in slow online learning further improvements develop over multiple sessions (Doyon and Benali, 2005). It is of note that the duration of both phases is highly dependent on task complexity (Dayan and Cohen, 2011). Procedural consolidation occurs after practice and incorporates two distinct processes offline-improvement and memory stabilization. Offline-improvement refers to in-between training session skill improvement. Memory stabilization results in diminished interference in memory encoding or retrieval by another consecutive task (Robertson et al., 2004a). Long-term retention describes the skill retention after a prolonged (e.g., 1 year) post-training time interval (Romano et al., 2010). An additional interesting concept is the term savings. First described in the motor adaptation literature, it is defined as the impact of previous learning on faster retraining in consecutive sessions (Landi et al., 2011).

The addressed learning components are represented by specific neuroanatomical substrates. Increased activity of the premotor cortex, supplementary motor area (SMA), parietal regions, striatum, and the cerebellum and decreased activity in the dorsolateral prefrontal cortex (DLPFC), primary motor cortex (M1), and presupplementary motor area (pre-SMA) have been associated with the fast learning progresses. Whereas, increased activation in primary motor cortex (M1), primary somatosensory cortex, SMA, and putamen and decreased activation in lobule VI of the cerebellum have been implicated with the slow learning process (Floyer-Lea and Matthews, 2005; Dayan and Cohen, 2011). Explicit learning has been shown to be sleep- and implicit learning time-dependent (Robertson et al., 2004b). The role of the M1 in offline learning has been disentangled with inhibitory NIBS. Inhibitory repetitive transcranial magnetic stimulation (rTMS) of M1 interfered with early consolidation (Muellbacher et al., 2002), but not with over-night improvements of a motor practice

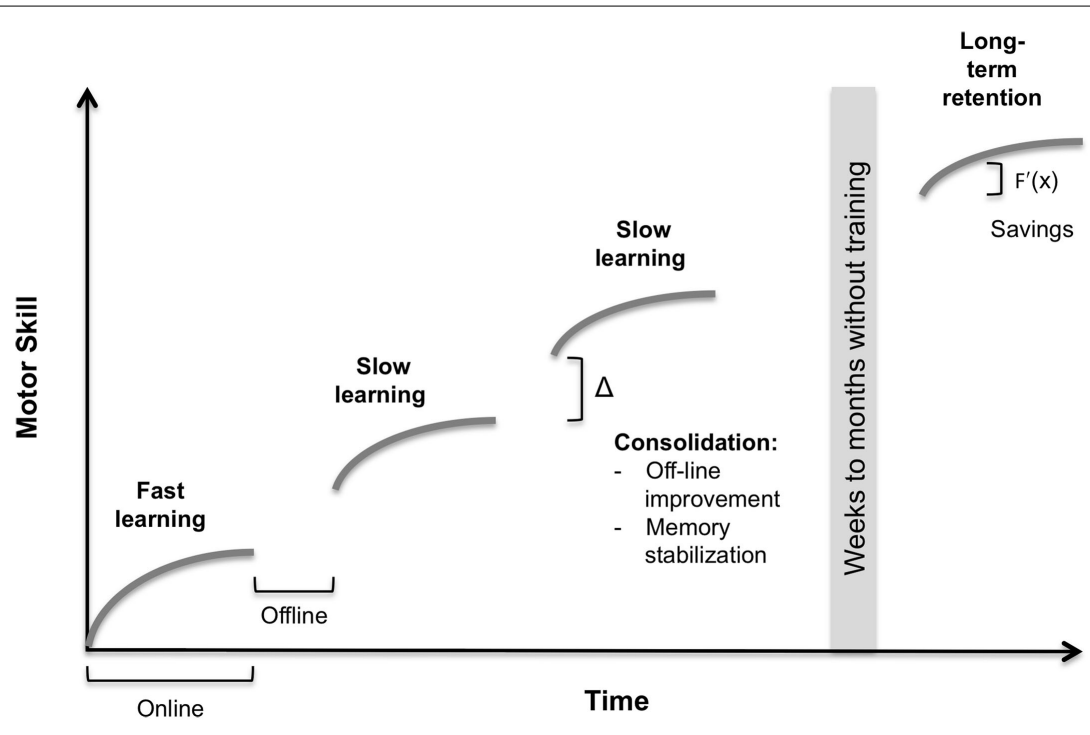

FIGURE 1 | Conceptual components in motor learning. The illustrated learning curve indicates the increase in motor skill over time.

Online - within-session learning. Offline - in-between-session learning. Fast learning - single-session practice. Slow learning - multiple-session practice.
Consolidation - offline-improvement and memory stabilization. Long-term retention - skill retention after a prolonged interval. Savings - impact of previous learning on faster retraining, expressed in an increased slope $f^{\prime}(x)$ of the learning curve. For a detailed description, please see text. 
(Robertson et al., 2005). However, sleep-dependent motor consolidation has been associated with increased activation in the striatum (Debas et al., 2010).

These neurobehavioral concepts depend on neuroplastic changes within the brain. Motor learning is incorporated in molecular, cellular, and systemic substrates. An association with de novo protein synthesis has been shown (Luft et al., 2004). Several cellular mechanisms, for instance, modulation of synaptic efficacy (Rioult-Pedotti et al., 2000), change in neuronal membrane excitability (Halter et al., 1995), and anatomical changes like spine formation (Greenough et al., 1985) and axonal sprouting (Toni et al., 1999), among others have been described. Moreover, during motor skill learning, changes in its cortical representation have been demonstrated (Pascual-Leone et al., 1995).

Also on the systems level, neuronal networks undergo plastic changes. Influential integrative models have been proposed (Hikosaka et al., 2002; Doyon and Benali, 2005). Doyon and Ungerleider hypothesize in their model that in early learning, cortico-striatal and cortico-cerebellar networks are recruited. Interactions of these structures shape the acquired motor memory traces during the learning process (Ungerleider et al., 2002; Doyon et al., 2003).

\section{Neuroplastic Changes Observed After Stroke}

Neuroplasticity after stroke and motor learning in the healthy brain share several common mechanisms. Described common neurobiological phenomena are axonal sprouting (Carmichael et al., 2001), dendritic remodeling (Brown et al., 2010), and reorganization of motor maps (Nudo and Milliken, 1996), among others. Furthermore, animal models could prove that rehabilitative training can influence the reorganization in the adjacent intact cortex (Nudo et al., 1996). For review, please see Hosp and Luft (2011) and Hallett (2001).

The neural correlates of stroke recovery have been described in several cross-sectional and longitudinal studies using functional neuroimaging (fMRI and PET) and TMS (Johansen-Berg et al., 2002; Ward et al., 2003; Gerloff et al., 2006; Lotze et al., 2006; Grefkes et al., 2008). Most of these studies revealed abnormal patterns of activation/excitability immediately after stroke and during the recovery process. Movement of the affected hand elicit a bilateral neural recruitment within motor areas in patients with subcortical lesions, which cannot be found in healthy subjects or when patients move the unaffected hand (Ward et al., 2003). In this regard, patients with good functional outcomes demonstrated a more lateralized neural activation pattern, while those patients whose motor deficit remained more severe recruited motor areas in both hemispheres (Ward et al., 2004). The significance of more widespread neural activation within the motor network during motor performance of the affected hand is still under debate. As recently discussed (Hummel et al., 2008a), the notion that the unaffected hemisphere has a non-beneficial effect on the lesioned hemisphere and consecutive behavior does not apply to all groups of patients. This fact might relevantly depend on lesion location, time after stroke, size, and integrity of the corticospinal pathway, among other factors (Lotze et al., 2006; Bradnam et al., 2011).
Patients, who suffered a stroke, exhibit changes in motor cortical excitability in both hemispheres (Shimizu et al., 2002; Cicinelli et al., 2003). Moreover, abnormal levels of inter-hemispheric inhibition from the unaffected to the affected motor cortex, resulting in an imbalanced inter-hemispheric interaction influence the function of the paretic hand (Murase et al., 2004) [for details regarding this concept, please see Schulz et al. (2013), Hummel and Cohen (2006), and Nowak et al. (2009)].

Following the concept of imbalanced inter-hemispheric inhibition, two main strategies for NIBS have been proposed: (1) NIBS is used to inhibit the motor cortex of the unaffected (contralesional - cM1) hemisphere to reduce the abnormal inhibitory drive toward the lesioned hemisphere. (2) An alternative approach is to facilitate the motor cortex of the affected (ipsilesional - iM1) hemisphere (Hummel and Cohen, 2006).

Additionally, neuroimaging studies have shown that premotor areas of the affected hemisphere are commonly more active during hand movement with the paretic hand after stroke, suggesting that non-primary motor areas of the same hemisphere are utilized when M1 is affected (Gerloff et al., 2006).

\section{Techniques for Non-Invasive Brain Stimulation}

Non-invasive brain stimulation techniques can be used to influence cortical excitability, neuroplasticity, and behavior [for review, see Nitsche et al. (2008), Hummel and Cohen (2005)]. Excitatory or inhibitory plastic changes can be induced, depending on the used mode. Transcranial direct current stimulation (tDCS), transcranial magnetic stimulation (TMS), and paired associative stimulation (PAS) are the most common and widely used techniques (Hummel and Cohen, 2005).

By using tDCS small sub-threshold currents (1-2 mA) delivered via scalp electrodes are capable of influencing neuronal excitability by increasing or decreasing the respective transmembrane potentials. The induced changes are polarity specific. Anodal stimulation facilitates and cathodal stimulation inhibits motor cortex excitability (Nitsche et al., 2008). Advantages of tDCS are its simplicity and relative low cost. Limitations are its rather moderate temporal and focal resolution (Gandiga et al., 2006).

By adjusting the electrode placement (electrode montage), different cortical areas can be modulated. An active (target) electrode is placed over the target area and a reference (return) electrode over another cephalic or extracephalic region. The scalp position of electrode placement is usually defined by anatomical landmarks, the TMS hotspot, the 10/20 EEG system, or via stereotactical neuronavigation (Moos et al., 2012). It is important to note that the reference electrode is not physiologically inert (Nitsche et al., 2008). Recently, a framework for the categorization of tDCS montages has been proposed (Nasseri et al., 2015). Most tDCS studies in stroke rehabilitation so far used a bilateral bipolar nonbalanced or balanced montage. In addition, first modeling studies, using finite element realistic head models, were able to calculate cortical current density distributions considering brain lesions (Wagner et al., 2007) and multifocal stimulation (Ruffini et al., 2014). This could help to find revised montages for future studies. 
Transcranial magnetic stimulation uses the principle of electromagnetic induction. With a sufficient induced electrical field, it is possible to depolarize neurons. When the magnetic pulses are applied in a repetitive mode (rTMS), this results in excitability changes outlasting the duration of stimulation (Rossi et al., 2009). Conventional rTMS applied at a low frequency $(0.2-1 \mathrm{~Hz})$ results in inhibition. When it is applied at high frequency $(\geq 5 \mathrm{~Hz})$, it leads to excitation (Hallett, 2007). Recently, novel patterned rTMS protocols have been developed, the so-called theta-burst stimulation (TBS) (Huang et al., 2005). In the theta-burst protocol (TBS), three stimuli at $50 \mathrm{~Hz}$ are repeated at $5 \mathrm{~Hz}$. In a continuous mode, this results in inhibition, in an intermittent mode in excitation (Hallett, 2007). Advantages of rTMS are its good spatial and temporal resolution, disadvantages are the relative complex and expensive setup and the rare but apparent occasion of relevant side effects, in particular seizures (Gandiga et al., 2006).

Paired associative stimulation is capable of inducing heterosynaptic plasticity. This is achieved by combining low-frequency peripheral nerve stimulation (PNS) with TMS to the motor cortex. The underlying concept is based on the idea that if both stimuli arrive at the same time (synchronous) at the cortex, an excitation will be induced. By contrast, asynchronous stimulation leads to inhibition (Stefan et al., 2000). Repetitive synchronous stimulation results in LTP-like effects, asynchronous stimulation in LTD-like effects. Advantages of PAS are that the protocol was directly developed on the basis of LTP/LTD-plasticity protocols of basic research and that many of its physiological properties are well studied. Disadvantages are its rather complex setup, its interindividual variability, and that effects of protocol variation have not been systematically studied (Ziemann et al., 2008).

Recently, the repertoire of NIBS techniques has been enlarged by transcranial alternating current stimulation (tACS), transcranial random noise stimulation (tRNS), and more complex rTMS protocols (quadri- and octapulse rTMS). These methods have not been widely applied to enhance functional recovery after stroke, for detailed descriptions of these techniques, please see Antal and Paulus (2013), Terney et al. (2008), and Hamada and Ugawa (2010).

\section{Non-Invasive Brain Stimulation and Motor Learning in Healthy Subjects}

First studies in healthy volunteers provided evidence that NIBS can cause transient behavioral effects in motor function (Wassermann et al., 1996; Boggio et al., 2006). Following this concept, first studies were designed to investigate whether NIBS can also modulate motor learning. The main target of most studies conducted so far was M1. In a first study by Nitsche et al. (2003), anodal tDCS applied to the contralateral M1 improved learning of an implicit task (Nitsche et al., 2003). By using an isometric-pinch task, Reis et al. (2009b) were able to demonstrate improved learning after five consecutive days of training with tDCS compared to sham, primarily driven by an offline (consolidation) effect. tDCS not only led to significant greater total learning but also the behavioral improvement remained superior in the tDCS group compared to sham even up to 3 months after training (Reis et al., 2009b). Anodal tDCS applied concurrently with practice demonstrated to enhance encoding and retention of a motor memory on a shorter time-scale. More recently, Tecchio et al. (2010) reported an improvement of early motor memory consolidation accomplished by anodal tDCS over the M1 in a serial finger-tapping task (Tecchio et al., 2010). Overall, there is accumulative evidence that tDCS is effective in promoting long-term plastic changes associated with learning and memory formation when increased excitability and changes in synaptic efficacy co-occur. It is of note that the facilitatory effect of anodal tDCS on learning might be task specific, depending on the state of cortical activation induced by the motor task (Bortoletto et al., 2014).

Furthermore, PAS modulated plasticity in healthy young subjects. PAS was paired with rapid thumb abduction movements, a basic model of motor learning; this led to the prevention of $\mathrm{PAS}_{\mathrm{syn}^{-}}$ induced LTP-like plasticity. Moreover, when PAS asyn was paired with this basic motor learning paradigm, subsequent $\mathrm{PAS}_{\text {asyn }}{ }^{-}$ induced LTD-plasticity was enhanced (Ziemann et al., 2004). This provides evidence that PAS can induce use-dependent plasticity in healthy subjects and interacts with motor learning.

A revealing model to study altered motor learning networks is healthy aging. In a promising study, anodal tDCS applied to M1 concurrently to an explicit motor learning task resulted in substantial improvements during training (Zimerman et al., 2013). Furthermore, the anodal stimulation group showed superior performance at the 24-h follow-up compared to sham.

\section{Non-Invasive Brain Stimulation and Motor Learning in Stroke}

The promising results of the discussed conceptual studies in young and old healthy subjects led to the interesting hypothesis of testing NIBS as an adjuvant therapy in neurological disorders. A resulting question was whether transient behavioral effects could be reproduced in stroke patients. The main conceptual target was to use NIBS for normalizing imbalanced inter-hemispheric inhibition. Following proof-of-principle studies demonstrated that anodal tDCS applied to iM1 and cathodal tDCS applied to cM1 improved transiently motor performance of the affected upper limb (Fregni et al., 2005; Hummel et al., 2005, 2006). Complementary rTMSstudies revealed that modulation of transcallosal inhibition with inhibitory $1 \mathrm{~Hz}$ rTMS to cM1 (Mansur et al., 2005; Takeuchi et al., 2005) and excitatory $20 \mathrm{~Hz}$ rTMS to iM1 improved motor function in chronic stroke patients (Yozbatiran et al., 2009).

As discussed in greater detail above, motor learning is essential for neurorehabilitation (Krakauer, 2006). In this regard, a pivotal question was whether NIBS could facilitate motor learning in patients. First studies investigated the effect of NIBS on motor learning in chronic stroke patients. For instance, cathodal tDCS applied to the $\mathrm{cM} 1$ during the performance of explicit motor learning task enhanced fast online acquisition yielding to a better task retention after $24 \mathrm{~h}$. Interestingly, there was an association between $\mathrm{tDCS}$-induced improvement during training and GABAergic intracortical changes in the affected motor cortex (Zimerman et al., 2012). Moreover, it could be shown that excitatory $10 \mathrm{~Hz}$ rTMS applied to iM1 immediately before each block of a sequential finger-tapping task, enhanced its acquisition in chronic stroke patients (Kim et al., 2006). 
The main objective for translational research is to combine NIBS with repetitive occupational therapy, which uses principles of motor learning, in a clinical setting. For instance, Kim et al. paired 10 sessions of tDCS in a double-blind parallel design with occupational therapy of the upper limb in sub-acute stroke patients. Cathodal tDCS to cM1 resulted in superior motor function, assessed with the Fugl-Meyer Score, in a 6-month follow-up (Kim et al., 2010). In addition, excitatory rTMS to iM1 as add-on to normal physical therapy over 10 consecutive days in sub-acute stroke patients improved patients' motor scores when compared to sham (Khedr et al., 2005). Bolognini and collaborators paired bihemispheric tDCS (cathodal stimulation to $\mathrm{cM} 1$ an anodal stimulation to iM1) with constraint-induced movement therapy (CIMT) over 10 treatment sessions in chronic stroke patients. Patients in the active group showed superior gains in hand function, measured with the Jebsen Taylor Hand Function Test (JTT), Handgrip Strength, Motor Activity Log Scale, and Fugl-Meyer Motor Score. Beyond that, as a neurophysiological correlate, the authors could identify a reduction in inter-hemispheric inhibition from the intact to the affected hemisphere, measured with double-pulse TMS (Bolognini et al., 2011). Another interesting concept, especially for patients with severe upper limb paresis, is the combination of tDCS with robot-assisted arm training. However, first promising results of a pilot study from Hesse et al. (2007) could not be replicated in a subsequent larger trial (Hesse et al., 2011). A possible explanation could be that the majority of the recruited patients had large cortical lesions and were severely affected. In a secondary analysis, patients with pure subcortical lesions improved significantly more after cathodal stimulation of the cM1 than patients with cortical involvement, pointing toward a need of individual patient stratification.

Further evidence that lesion location might have a crucial impact on the response to stimulation comes from a study conducted by Ameli et al. (2009). In their study, patients with subcortical stroke improved in dexterity after $10 \mathrm{~Hz}$ rTMS to iM1. This beneficial effect was not apparent in patients with additional cortical strokes (Ameli et al., 2009).

A compelling approach is dual-tDCS stimulation (anodal electrode over iM1 and cathodal electrode over cM1). Lefebvre et al. (2012) used this montage in chronic stroke patients with moderate deficits while training a complex visuomotor skill. A singlesession of dual tDCS enhanced the online learning, leading to superior long-term retention (Lefebvre et al., 2012). Although promising, there is so far no clear scientific evidence that bifocal stimulation is more efficient than monofocal, or for which patients' bifocal or monofocal might lead to more improvements. These are important questions, which have to be addressed in upcoming larger trials. Recently, novel training regimes, like virtual reality motor training, have been paired with tDCS in subacute stroke patients and have shown beneficial effects (Kim et al., 2014a).

In addition, first studies evaluated the feasibility of PAS in stroke patients. $\mathrm{PAS}_{\text {syn }}$ resulted in a significant facilitation of the extensor carpi radialis (ECR) MEP amplitude of the paretic side after 5 months following subcortical stroke. Partly, the facilitation was still present 12 months after the stroke. Furthermore, the neurophysiological changes were accompanied with improvements in the wrist section of the Fugl-Meyer motor scale and wrist force (Castel-Lacanal et al., 2007, 2009).

To date, a number of sham-controlled studies have been performed to investigate stimulation-associated enhancement of motor recovery after stroke (for detailed overview, please see Table 1). To evaluate the potential beneficial effects of NIBS, further multicenter clinical trials are needed. In this regard, the NETS-trial (Neuroregeneration Enhanced by Transcranial Direct Current Stimulation (tDCS) in Stroke, ClinTrialGov NCT009097 14) has been initiated. In the on-going study, anodal tDCS to iM1 combined with standard occupational therapy is applied in 10 consecutive sessions in sub-acute stroke patients.

\section{Potential Strategies and Underling Mechanisms to Increase the Effect of Non-Invasive Brain Stimulation}

Effect sizes in the range between 8 and $30 \%$ of functional improvement in stroke patients have been reported for NIBS (Hummel et al., 2008b). In the next section, underlying principles and strategies to increase the effectivity are discussed (for illustration, see Figure 2; Table 2).

\section{Where to Stimulate?}

Most studies conducted so far have focused on motor cortical stimulation. Both the iM1 and cM1 have been evaluated as targets. The advantage of stimulating iM1 is the possibility to directly enhance its reduced participation in the incompletely recovered motor network after stroke (Hummel et al., 2008b). Disadvantages are that the lesioned motor cortex might be more prone to adverse effects, for example, induction of excitotoxicity in the penumbra in the acute phase and the shunting of electrical current. Advantage of stimulating the $\mathrm{cM} 1$ is that NIBS is applied to intact cortical areas, especially relevant in patients with large almost complete lesions of iM1, thus the cM1 could here be used as a "window" to influence the motor system (Hummel et al., 2008b). A disadvantage is that inhibitory stimulation might also impair complex motor function of the paretic and the intact hand (Lotze et al., 2006). An alternative approach could be the stimulation of secondary sensorimotor areas, basal ganglia and cerebellum; for a conceptual review, please see Plow et al. (2014). First proof-of-principle studies provided promising results for SMA (Vollmann et al., 2013) and cerebellar (Galea et al., 2011; Wessel et al., 2015) stimulation combined with motor learning paradigms in healthy volunteers. In addition, recent studies demonstrated that inhibitory rTMS stimulation over cPMd (Wang et al., 2014) and the cerebellum (Kim et al., 2014b) improved motor function in stroke patients. Furthermore, excitatory rTMS over the ipsilesional primary sensory cortex (iS1) in chronic stroke facilitated learning of a Serial Tracking Task (Brodie et al., 2014).

\section{When to Stimulate?}

In addition, the timing of the stimulation is most likely crucial for efficacy in motor rehabilitation. Since NIBS and motor learning 


$\begin{array}{ccccccccc}\begin{array}{c}\text { Number } \\ \text { of } \\ \text { patients }\end{array} & \begin{array}{c}\text { Cortical/ } \\ \text { subcortical }\end{array} & \begin{array}{c}\text { Ischemic/ } \\ \text { hemorrhagic }\end{array} & \begin{array}{c}\text { Severity } \\ \text { of stroke }\end{array} & \begin{array}{c}\text { Stroke } \\ \text { duration }\end{array} & \begin{array}{c}\text { Motor } \\ \text { assessments } \\ \text { and outcomes }\end{array} & \begin{array}{c}\text { Concomitant } \\ \text { therapy }\end{array} & \begin{array}{c}\text { Study } \\ \text { design }\end{array} & \begin{array}{c}\text { NIBS } \\ \text { Intervention } \\ \text { sessions }\end{array}\end{array}$

\section{rTMS}

\begin{tabular}{|c|c|c|c|c|c|c|c|c|c|c|c|c|}
\hline $\begin{array}{l}\text { Khedr et al. } \\
\text { (2005) }\end{array}$ & 52 & $\begin{array}{l}\text { Cortico- } \\
\text { subcortical }\end{array}$ & Ischemic & $\begin{array}{l}\text { Moderate to } \\
\text { severe }\end{array}$ & 5-10 days & SSS, NIHSS, BI & $\begin{array}{l}\text { Standard } \\
\text { physical therapy }\end{array}$ & $\begin{array}{l}\text { Randomized, } \\
\text { parallel groups }\end{array}$ & $\begin{array}{l}3 \mathrm{~Hz} \text { rTMS over } \\
\text { iM1 }\end{array}$ & 10 days & 10 days & Pos \\
\hline $\begin{array}{l}\text { Mansur et al. } \\
(2005)\end{array}$ & $\begin{array}{l}\text { Stroke } 10 \text {, } \\
\text { healthy } 6\end{array}$ & $\begin{array}{l}\text { Cortico- } \\
\text { subcortical }\end{array}$ & Ischemic & Mixed & $<12$ months & sRT, cRT, PP, FT & NA & $\begin{array}{l}\text { Randomized, } \\
\text { cross-over }\end{array}$ & $\begin{array}{l}1 \mathrm{~Hz} \text { rTMS over } \\
\mathrm{cM} 1 \text { and cPM }\end{array}$ & One & NA & Pos \\
\hline $\begin{array}{l}\text { Takeuchi } \\
\text { et al. (2005) }\end{array}$ & 20 & Subcortical & Ischemic & Mixed & 26.95 months & FM, PA & NA & $\begin{array}{l}\text { Randomized, } \\
\text { parallel groups }\end{array}$ & $\begin{array}{l}1 \mathrm{~Hz} \text { rTMS over } \\
\mathrm{cM} 1\end{array}$ & One & NA & Pos \\
\hline $\begin{array}{l}\text { Fregni et al. } \\
(2006)\end{array}$ & 15 & $\begin{array}{l}\text { Cortical } 1 \text {, } \\
\text { subcortical } 13 \text {, } \\
\text { cortico- } \\
\text { subcortical } 1\end{array}$ & Ischemic & $\begin{array}{l}\text { Mild to } \\
\text { moderate }\end{array}$ & 44.05 months & $\begin{array}{l}\text { MRC, ASS, JTT, } \\
\text { sRT, cRT, PPT }\end{array}$ & NA & $\begin{array}{l}\text { Randomized, } \\
\text { parallel groups }\end{array}$ & $\begin{array}{l}\text { 1 Hz rTMS over } \\
\mathrm{cM} 1\end{array}$ & 5 days & 2 weeks & Pos \\
\hline $\begin{array}{l}\text { Kim et al. } \\
(2006)\end{array}$ & 15 & $\begin{array}{l}\text { Cortical } 5 \\
\text { subcortical } 10\end{array}$ & $\begin{array}{l}\text { Ischemic } 12 \text {, } \\
\text { hemorrhagic } 3\end{array}$ & $\begin{array}{l}\text { Mild to } \\
\text { moderate }\end{array}$ & 16.7 months & PP, GF, FT & $\mathrm{FT}$ & $\begin{array}{l}\text { Randomized, } \\
\text { cross-over }\end{array}$ & $\begin{array}{l}10 \mathrm{~Hz} \text { rTMS } \\
\text { over iM1 }\end{array}$ & One & NA & Pos \\
\hline $\begin{array}{l}\text { Liepert et al. } \\
(2007)\end{array}$ & 12 & $\begin{array}{l}\text { Subcortical (2 } \\
\text { pons) }\end{array}$ & NM & Mild & 7.3 days & MRC, GF, NHPT & NA & $\begin{array}{l}\text { Randomized, } \\
\text { cross-over }\end{array}$ & $\begin{array}{l}1 \mathrm{~Hz} \text { rTMS over } \\
\mathrm{cM} 1\end{array}$ & One & NA & Pos \\
\hline $\begin{array}{l}\text { Malcolm } \\
\text { et al. (2007) }\end{array}$ & 20 & $\begin{array}{l}\text { Cortico- } \\
\text { subcortical }\end{array}$ & Hemorrhagic 1 & Mixed & 45.6 months & WMFT, BBT, MAL & CIT & $\begin{array}{l}\text { Randomized, } \\
\text { parallel groups }\end{array}$ & $\begin{array}{l}20 \mathrm{~Hz} \text { rTMS } \\
\text { over iM1 }\end{array}$ & 10 days & 6 months & Neg \\
\hline $\begin{array}{l}\text { Talelli et al. } \\
\text { (2007) }\end{array}$ & 6 & $\begin{array}{l}\text { Cortical 3, } \\
\text { subcortical } 3\end{array}$ & Ischemic & $\begin{array}{l}\text { Mild to } \\
\text { moderate }\end{array}$ & 31 months & $\begin{array}{l}\text { BI, NIHSS, ARAT, } \\
\text { 9HP, GF, sRT, cRT }\end{array}$ & NA & $\begin{array}{l}\text { Randomized, } \\
\text { cross-over }\end{array}$ & $\begin{array}{l}\text { iTBS over iM1, } \\
\text { cTBS over cM1 }\end{array}$ & One & NA & Pos \\
\hline $\begin{array}{l}\text { Dafotakis } \\
\text { et al. (2008) }\end{array}$ & 12 & Subcortical & Ischemic & Mild & 1.88 months & $\begin{array}{l}\text { MRC (4-5), NIHSS, } \\
\text { ARAT, grip-lift task }\end{array}$ & $\begin{array}{l}\text { Grasping and } \\
\text { lifting }\end{array}$ & $\begin{array}{l}\text { Randomized, } \\
\text { cross-over }\end{array}$ & $\begin{array}{l}1 \mathrm{~Hz} \text { rTMS over } \\
\mathrm{cM} 1\end{array}$ & One & NA & Pos \\
\hline $\begin{array}{l}\text { Mally and } \\
\text { Dinya (2008) }\end{array}$ & 64 & Cortical, large & $\begin{array}{l}\text { Ischemic, } \\
\text { hemorrhagic } 18\end{array}$ & Severe & 129.6 months & Spasticity score & NA & $\begin{array}{l}\text { Randomized, } \\
\text { parallel groups }\end{array}$ & $\begin{array}{l}\text { 1 Hz rTMS over } \\
\text { cM1 and iM1 }\end{array}$ & 7 days & 3 months & Pos \\
\hline $\begin{array}{l}\text { Nowak et al. } \\
(2008)\end{array}$ & 15 & Subcortical & Ischemic & Mild & 1.93 months & $\begin{array}{l}\text { ARAT, MRC (4-5), } \\
\mathrm{FT} \text {, reach to grasp }\end{array}$ & NA & $\begin{array}{l}\text { Randomized, } \\
\text { cross-over }\end{array}$ & $\begin{array}{l}1 \mathrm{~Hz} \text { rTMS over } \\
\mathrm{cM} 1\end{array}$ & One & NA & Pos \\
\hline $\begin{array}{l}\text { Takeuchi } \\
\text { et al. (2008) }\end{array}$ & 20 & Subcortical & Ischemic & Mixed & 29.9 months & $\begin{array}{l}\mathrm{FM} \text {, acceleration } \\
\text { and PF }\end{array}$ & PF training & $\begin{array}{l}\text { Randomized, } \\
\text { parallel groups }\end{array}$ & $\begin{array}{l}1 \mathrm{~Hz} \text { rTMS over } \\
\mathrm{cM} 1\end{array}$ & One & 1 week & Pos \\
\hline $\begin{array}{l}\text { Ameli et al. } \\
\text { (2009) }\end{array}$ & 29 & $\begin{array}{l}\text { Cortical } 13 \\
\text { subcortical } 16\end{array}$ & Ischemic & $\begin{array}{l}\text { Mild to } \\
\text { moderate }\end{array}$ & 5.5 months & $\begin{array}{l}\text { MRC, ARAT, mRS, } \\
\text { NIHSS, index finger } \\
\text { and hand tapping }\end{array}$ & NA & $\begin{array}{l}\text { Randomized, } \\
\text { cross-over }\end{array}$ & $\begin{array}{l}10 \mathrm{~Hz} \text { rTMS } \\
\text { over iM1 }\end{array}$ & One & NA & Mix \\
\hline $\begin{array}{l}\text { Khedr et al. } \\
\text { (2009) }\end{array}$ & 36 & $\begin{array}{l}\text { Cortical } 19 \\
\text { subcortical } 17\end{array}$ & Ischemic & $\begin{array}{l}\text { Mild to } \\
\text { moderate }\end{array}$ & 0.57 months & $\begin{array}{l}\text { MRC, NIHSS, BI, } \\
\text { tapping, PP }\end{array}$ & NA & $\begin{array}{l}\text { Randomized, } \\
\text { parallel groups }\end{array}$ & $\begin{array}{l}1 \mathrm{~Hz} \text { rTMS over } \\
\text { cM1, 3 Hz over } \\
\text { iM1 }\end{array}$ & Five & 3 months & Pos \\
\hline $\begin{array}{l}\text { Takeuchi } \\
\text { et al. (2009) }\end{array}$ & 30 & Subcortical & Ischemic & Mixed & 28.8 months & $\begin{array}{l}\text { FM, acceleration } \\
\text { and PF }\end{array}$ & $\begin{array}{l}\text { Motor training } \\
\text { (pinching task) }\end{array}$ & $\begin{array}{l}\text { Randomized, } \\
\text { parallel groups }\end{array}$ & $\begin{array}{l}1 \mathrm{~Hz} \text { rTMS over } \\
\mathrm{cM} 1,10 \mathrm{~Hz} \text { over } \\
\text { iM1, bilateral } \\
\text { rTMS }\end{array}$ & One & 1 week & Pos \\
\hline $\begin{array}{l}\text { Chang et al. } \\
\text { (2010) }\end{array}$ & 28 & $\begin{array}{l}\text { Cortical } 11 \\
\text { subcortical } 17\end{array}$ & NM & $\begin{array}{l}\text { Moderate to } \\
\text { severe }\end{array}$ & 13.4 days & Ml, FM, GF, BB & $\begin{array}{l}\text { Reaching and } \\
\text { grasping } \\
\text { exercises }\end{array}$ & $\begin{array}{l}\text { Randomized, } \\
\text { parallel groups }\end{array}$ & $10 \mathrm{~Hz}$ over iM1 & 10 & 3 months & Pos \\
\hline
\end{tabular}


TABLE 1 | Continued

\begin{tabular}{|c|c|c|c|c|c|c|c|c|c|c|c|c|}
\hline & $\begin{array}{c}\text { Number } \\
\text { of } \\
\text { patients }\end{array}$ & $\begin{array}{l}\text { Cortical/ } \\
\text { subcortical }\end{array}$ & $\begin{array}{c}\text { Ischemic/ } \\
\text { hemorrhagic }\end{array}$ & $\begin{array}{l}\text { Severity } \\
\text { of stroke }\end{array}$ & $\begin{array}{c}\text { Stroke } \\
\text { duration }\end{array}$ & $\begin{array}{c}\text { Motor } \\
\text { assessments } \\
\text { and outcomes }\end{array}$ & $\begin{array}{l}\text { Concomitant } \\
\text { therapy }\end{array}$ & $\begin{array}{l}\text { Study } \\
\text { design }\end{array}$ & $\begin{array}{c}\text { NIBS } \\
\text { Intervention }\end{array}$ & $\begin{array}{c}\text { Number } \\
\text { of } \\
\text { sessions }\end{array}$ & Follow-ups & Result \\
\hline $\begin{array}{l}\text { Emara et al. } \\
\text { (2010) }\end{array}$ & 60 & $\begin{array}{l}\text { Cortical } 43 \\
\text { subcortical } 17\end{array}$ & Ischemic & $\begin{array}{l}\text { Mild to } \\
\text { moderate }\end{array}$ & $>1$ month & $\mathrm{FT}, \mathrm{mRS}, \mathrm{Al}$ & $\begin{array}{l}\text { Standard } \\
\text { physical therapy }\end{array}$ & $\begin{array}{l}\text { Randomized, } \\
\text { parallel groups }\end{array}$ & $\begin{array}{l}5 \mathrm{~Hz} \text { rTMS over } \\
\mathrm{iM} 1,1 \mathrm{~Hz} \text { rTMS } \\
\text { over cM1 }\end{array}$ & Ten & 12 weeks & Pos \\
\hline $\begin{array}{l}\text { Grefkes et al. } \\
\text { (2010) }\end{array}$ & 11 & Subcortical & Ischemic & Mild & 1.91 months & $\begin{array}{l}\text { MRC (4-5), ARAT, } \\
\text { NIHSS, whole hand } \\
\text { fist task }\end{array}$ & NA & $\begin{array}{l}\text { Randomized, } \\
\text { cross-over }\end{array}$ & $\begin{array}{l}1 \mathrm{~Hz} \text { rTMS over } \\
\mathrm{cM} 1\end{array}$ & One & NA & Pos \\
\hline $\begin{array}{l}\text { Avenanti } \\
\text { et al. (2012) }\end{array}$ & 30 & $\begin{array}{l}\text { Cortical } 3 \text {, } \\
\text { cortico- } \\
\text { subcortical } 1 \text {, } \\
\text { subcortical } 26\end{array}$ & $\begin{array}{l}\text { Ischemic 20, } \\
\text { hemorrhagic } 10\end{array}$ & Mild & 31.47 months & JTT, NHPT, BB, PF & $\begin{array}{l}\text { Standard } \\
\text { physical therapy }\end{array}$ & $\begin{array}{l}\text { Randomized, } \\
\text { parallel groups }\end{array}$ & $\begin{array}{l}1 \mathrm{~Hz} \text { rTMS over } \\
\mathrm{cM} 1\end{array}$ & 10 & 3 months & Pos \\
\hline $\begin{array}{l}\text { Chang et al. } \\
\text { (2012) }\end{array}$ & 17 & $\begin{array}{l}\text { Cortical 2, } \\
\text { subcortical } 15\end{array}$ & $\begin{array}{l}\text { Ischemic } 14 \text {, } \\
\text { hemorrhagic } 3\end{array}$ & $\begin{array}{l}\text { Mild to } \\
\text { moderate }\end{array}$ & $>3$ months & JTT, SFIT & SFIT & $\begin{array}{l}\text { Randomized, } \\
\text { parallel groups }\end{array}$ & $\begin{array}{l}10 \mathrm{~Hz} \text { rTMS } \\
\text { over iM1 }\end{array}$ & 10 & 1 month & Pos \\
\hline $\begin{array}{l}\text { Conforto } \\
\text { et al. (2012) }\end{array}$ & 30 & $\begin{array}{l}\text { Subcortical 16, } \\
\text { cortical } 14\end{array}$ & Ischemic & $\begin{array}{l}\text { Mild to } \\
\text { severe }\end{array}$ & 0.92 months & $\begin{array}{l}\text { MRC, NIHSS, JTT, } \\
\text { PF }\end{array}$ & $\begin{array}{l}\text { Standard } \\
\text { physical therapy }\end{array}$ & $\begin{array}{l}\text { Randomized, } \\
\text { parallel groups }\end{array}$ & $\begin{array}{l}1 \mathrm{~Hz} \text { rTMS, over } \\
\mathrm{cM} 1\end{array}$ & 10 & 1 month & Pos \\
\hline $\begin{array}{l}\text { Seniow et al. } \\
\text { (2012) }\end{array}$ & 40 & $\begin{array}{l}\text { Cortical 16, } \\
\text { subcortical } 14\end{array}$ & $\begin{array}{l}\text { Ischemic } 35 \text {, } \\
\text { hemorrhagic } 5\end{array}$ & Moderate & $<3$ months & WMFT, NIHSS, FM & $\begin{array}{l}\text { Standard } \\
\text { physical therapy }\end{array}$ & $\begin{array}{l}\text { Randomized, } \\
\text { parallel groups }\end{array}$ & $1 \mathrm{~Hz}$ over $\mathrm{cM} 1$ & 3 weeks & 3 months & Neg \\
\hline $\begin{array}{l}\text { Wang et al. } \\
\text { (2012) }\end{array}$ & 28 & NM & NM & Moderate & $>6$ months & FM, WP & $\begin{array}{l}\text { Task-oriented } \\
\text { training }\end{array}$ & $\begin{array}{l}\text { Randomized, } \\
\text { parallel groups }\end{array}$ & $\begin{array}{l}1 \mathrm{~Hz} \text { rTMS over } \\
\mathrm{cM} 1\end{array}$ & Ten & NA & Pos \\
\hline $\begin{array}{l}\text { Etoh et al. } \\
\text { (2013) }\end{array}$ & 18 & $\begin{array}{l}\text { Cortical } 1 \text {, } \\
\text { subcortical } 17\end{array}$ & $\begin{array}{l}\text { Ischemic } 13 \text {, } \\
\text { hemorrhagic } 5\end{array}$ & Severe & 29.9 months & FM, ARAT, MAS & $\begin{array}{l}\text { Physical or } \\
\text { occupational } \\
\text { therapy }\end{array}$ & $\begin{array}{l}\text { Randomized, } \\
\text { cross-over }\end{array}$ & $\begin{array}{l}1 \mathrm{~Hz} \text { rTMS over } \\
\mathrm{cM} 1\end{array}$ & Ten & 4 weeks & Pos \\
\hline $\begin{array}{l}\text { Higgins et al. } \\
\text { (2013) }\end{array}$ & 11 & NM & NM & $\begin{array}{l}\text { Mild to } \\
\text { severe }\end{array}$ & $>3$ months & $\begin{array}{l}\text { BB, WMFT, MAL, } \\
\text { GF, PF, SIS }\end{array}$ & $\begin{array}{l}\text { Task-Oriented } \\
\text { Training }\end{array}$ & $\begin{array}{l}\text { Randomized, } \\
\text { parallel groups }\end{array}$ & $\begin{array}{l}1 \mathrm{~Hz} \text { TMS over } \\
\mathrm{cM} 1\end{array}$ & Eight & NA & Neg \\
\hline $\begin{array}{l}\text { Sasaki et al. } \\
\text { (2013) }\end{array}$ & 29 & Subcortical & $\begin{array}{l}\text { Ischemic 13, } \\
\text { hemorrhagic } 16\end{array}$ & $\begin{array}{l}\text { Mild to } \\
\text { moderate }\end{array}$ & 0.58 months & NIHSS, GF, FT & $\begin{array}{l}\text { Standard } \\
\text { physical therapy }\end{array}$ & $\begin{array}{l}\text { Randomized, } \\
\text { parallel groups }\end{array}$ & $\begin{array}{l}10 \mathrm{~Hz} \text { rTMS } \\
\text { over iM1, } 1 \mathrm{~Hz} \\
\text { rTMS over cM1 }\end{array}$ & Five & NA & Pos \\
\hline $\begin{array}{l}\text { Sung et al. } \\
\text { (2013) }\end{array}$ & 54 & $\begin{array}{l}\text { Cortical 35, } \\
\text { subcortical } 19\end{array}$ & $\begin{array}{l}\text { Ischemic 35, } \\
\text { hemorrhagic } 19\end{array}$ & Severe & 3-12 months & GF, FM, WMST, RT & NA & $\begin{array}{l}\text { Randomized, } \\
\text { parallel groups }\end{array}$ & $\begin{array}{l}1 \mathrm{~Hz} \text { rTMS over } \\
\text { cM1/TTBS over } \\
\text { iM1 }\end{array}$ & 20 & NA & Pos \\
\hline $\begin{array}{l}\text { Brodie et al. } \\
\text { (2014) }\end{array}$ & 15 & $\begin{array}{l}\text { Cortico- } \\
\text { subcortical 5, } \\
\text { subcortical } 9\end{array}$ & NM & $\begin{array}{l}\text { Mild to } \\
\text { moderate }\end{array}$ & $>6$ months & $\begin{array}{l}\text { STT, 2PD, WMFT, } \\
\text { BB, GF }\end{array}$ & STT & $\begin{array}{l}\text { Randomized, } \\
\text { parallel groups }\end{array}$ & $\begin{array}{l}5 \mathrm{~Hz} \text { rTMS over } \\
\text { is1 }\end{array}$ & Five & 1 day & Mix \\
\hline $\begin{array}{l}\text { Rose et al. } \\
\text { (2014) }\end{array}$ & 19 & NM & Ischemic & Moderate & $>6$ months & $\begin{array}{l}\text { WMFT, FM, ARAT, } \\
\text { GF, PF, MAS, MAL }\end{array}$ & $\begin{array}{l}\text { Functional Task } \\
\text { Practice }\end{array}$ & $\begin{array}{l}\text { Randomized, } \\
\text { parallel groups }\end{array}$ & $\begin{array}{l}\text { 1 Hz rTMS over } \\
\text { cM1 }\end{array}$ & 16 & 30 days & Neg \\
\hline $\begin{array}{l}\text { Motamed } \\
\text { Vaziri et al. } \\
\text { (2014) }\end{array}$ & 12 & NM & NM & Severe & $>2$ months & $\mathrm{BI}, \mathrm{FM}$ & $\begin{array}{l}\text { Standard } \\
\text { physical therapy }\end{array}$ & $\begin{array}{l}\text { Randomized, } \\
\text { parallel groups }\end{array}$ & $\begin{array}{l}1 \mathrm{~Hz} \text { rTMS over } \\
\mathrm{cM} 1\end{array}$ & 10 & NA & Pos \\
\hline $\begin{array}{l}\text { Wang et al. } \\
\text { (2014) }\end{array}$ & 44 & $\begin{array}{l}\text { Cortical 16, } \\
\text { subcortical } 28\end{array}$ & $\begin{array}{l}\text { Ischemic 29, } \\
\text { hemorrhagic } 15\end{array}$ & $\begin{array}{l}\text { Moderate to } \\
\text { severe }\end{array}$ & 3-12 months & MRC, FM, WMFT & $\begin{array}{l}\text { Standard } \\
\text { physical and } \\
\text { occupational } \\
\text { therapy }\end{array}$ & $\begin{array}{l}\text { Randomized, } \\
\text { parallel groups }\end{array}$ & $\begin{array}{l}1 \mathrm{~Hz} \text { rTMS over } \\
\mathrm{cM} 1,1 \mathrm{~Hz} \text { rTMS } \\
\text { over cPMd }\end{array}$ & 10 & NA & Pos \\
\hline
\end{tabular}


TABLE 1 | Continued

\begin{tabular}{|c|c|c|c|c|c|c|c|c|c|c|c|c|}
\hline & $\begin{array}{c}\text { Number } \\
\text { of } \\
\text { patients }\end{array}$ & $\begin{array}{l}\text { Cortical/ } \\
\text { subcortical }\end{array}$ & $\begin{array}{c}\text { Ischemic/ } \\
\text { hemorrhagic }\end{array}$ & $\begin{array}{l}\text { Severity } \\
\text { of stroke }\end{array}$ & $\begin{array}{l}\text { Stroke } \\
\text { duration }\end{array}$ & $\begin{array}{c}\text { Motor } \\
\text { assessments } \\
\text { and outcomes }\end{array}$ & $\begin{array}{c}\text { Concomitant } \\
\text { therapy }\end{array}$ & $\begin{array}{l}\text { Study } \\
\text { design }\end{array}$ & $\begin{array}{c}\text { NIBS } \\
\text { Intervention }\end{array}$ & $\begin{array}{l}\text { Number } \\
\text { of } \\
\text { sessions }\end{array}$ & Follow-ups & Result \\
\hline \multicolumn{13}{|l|}{ tDCs } \\
\hline $\begin{array}{l}\text { Fregni et al. } \\
\text { (2005) }\end{array}$ & 6 & $\begin{array}{l}\text { Cortico- } \\
\text { subcortical }\end{array}$ & NM & $\begin{array}{l}\text { Mild to } \\
\text { moderate }\end{array}$ & 27.1 months & MRC, ASS, JTT & NA & $\begin{array}{l}\text { Randomized, } \\
\text { double-blinded, } \\
\text { cross-over }\end{array}$ & $\begin{array}{l}\text { ctDCS over cM1 } \\
\text { and atDCS over } \\
\text { iM1 }\end{array}$ & One & NA & Pos \\
\hline $\begin{array}{l}\text { Hummel } \\
\text { et al. (2005) }\end{array}$ & 6 & $\begin{array}{l}\text { Subcortical 5, } \\
\text { cortico- } \\
\text { subcortical } 1\end{array}$ & Ischemic & Mild & 44.4 months & $\begin{array}{l}\text { MRC, FM, ASS, } \\
\text { JTT }\end{array}$ & NA & $\begin{array}{l}\text { Randomized, } \\
\text { double-blinded, } \\
\text { cross-over }\end{array}$ & atDCS over iM1 & One & 10 days & Pos \\
\hline $\begin{array}{l}\text { Hummel } \\
\text { et al. (2006) }\end{array}$ & 11 & Subcortical & Ischemic & $\begin{array}{l}\text { Mild to } \\
\text { moderate }\end{array}$ & 41.8 months & $\begin{array}{l}\text { MRC, FM, ASS, } \\
\text { sRT, PF }\end{array}$ & NA & $\begin{array}{l}\text { Randomized, } \\
\text { double-blinded, } \\
\text { cross-over }\end{array}$ & atDCS over iM1 & One & NA & Pos \\
\hline $\begin{array}{l}\text { Boggio et al. } \\
(2007)\end{array}$ & 9 & Subcortical & NA & $\begin{array}{l}\text { Mild to } \\
\text { moderate }\end{array}$ & 40.9 months & MRC, JTT, ASS & NA & $\begin{array}{l}\text { Randomized, } \\
\text { double-blinded, } \\
\text { cross-over }\end{array}$ & $\begin{array}{l}\text { ctDCS over CM1 } \\
\text { and atDCS over } \\
\text { iM1 }\end{array}$ & 5 days & 2 weeks & Pos \\
\hline $\begin{array}{l}\text { Kim et al. } \\
(2010)\end{array}$ & 18 & $\begin{array}{l}\text { Cortical 5, } \\
\text { subcortical 9, } \\
\text { cortico- } \\
\text { subcortical } 4\end{array}$ & Ischemic & Mixed & 25.6 days & $\begin{array}{l}\text { MRC (2-5) and FM } \\
(16-60), F M, B I\end{array}$ & $\begin{array}{l}\text { Occupational } \\
\text { therapy }\end{array}$ & $\begin{array}{l}\text { Randomized, } \\
\text { parallel groups }\end{array}$ & $\begin{array}{l}\text { ctDCS over } \\
\text { cM1, atDCS } \\
\text { over iM1 }\end{array}$ & 10 & 6 months & Mix \\
\hline $\begin{array}{l}\text { Lindenberg } \\
\text { et al. (2010) }\end{array}$ & 20 & $\begin{array}{l}\text { Cortico- } \\
\text { subcortical }\end{array}$ & Ischemic & Severe & 35.4 months & FM (20-56), WMF & $\begin{array}{l}\text { Occupational } \\
\text { therapy }\end{array}$ & $\begin{array}{l}\text { Randomized, } \\
\text { parallel groups }\end{array}$ & Bilateral tDCS & Five & 1 week & Pos \\
\hline $\begin{array}{l}\text { Bolognini } \\
\text { et al. (2011) }\end{array}$ & 14 & $\begin{array}{l}\text { Cortical 9, } \\
\text { cortico- } \\
\text { subcortical } 5\end{array}$ & $\begin{array}{l}\text { Ischemic 12, } \\
\text { hemorrhagic } 2\end{array}$ & $\begin{array}{l}\text { Moderate to } \\
\text { severe }\end{array}$ & 35.21 months & $\begin{array}{l}\text { FM, BI, JTT, HG, } \\
\text { MAL }\end{array}$ & CIT & $\begin{array}{l}\text { Randomized, } \\
\text { parallel groups }\end{array}$ & Bilateral tDCS & 10 & 4 weeks & Pos \\
\hline $\begin{array}{l}\text { Hesse et al. } \\
\text { (2011) }\end{array}$ & 96 & $\begin{array}{l}\text { Mixed cortico- } \\
\text { subcortical }\end{array}$ & Ischemic & Severe & 0.93 months & $\begin{array}{l}\mathrm{BI}, \mathrm{FM}(<18), \mathrm{BB} \\
\mathrm{MAS}, \mathrm{MRC}\end{array}$ & $\begin{array}{l}\text { Robot-assisted } \\
\text { arm training }\end{array}$ & $\begin{array}{l}\text { Randomized, } \\
\text { parallel groups, } \\
\text { multicenter }\end{array}$ & $\begin{array}{l}\text { ctDCS over CM1 } \\
\text { and atDCS over } \\
\text { iM1 }\end{array}$ & 30 (6 weeks) & 3 months & Neg \\
\hline $\begin{array}{l}\text { Madhavan } \\
\text { and Stinear } \\
\text { (2011) }\end{array}$ & 9 & $\begin{array}{l}\text { Cortico- } \\
\text { subcortical }\end{array}$ & NA & $\begin{array}{l}\text { Lower } \\
\text { extremity }\end{array}$ & 130.8 months & $\begin{array}{l}\text { FM-LE, dorsiflexion } \\
\text { and plantar flexion } \\
\text { movements }\end{array}$ & $\begin{array}{l}\text { Tracking } \\
\text { dorsiflexion and } \\
\text { plantar flexion } \\
\text { task }\end{array}$ & $\begin{array}{l}\text { Randomized, } \\
\text { cross-over }\end{array}$ & $\begin{array}{l}\text { atDCS over iM1, } \\
\text { atDCS over cM1 }\end{array}$ & One & NA & Pos \\
\hline $\begin{array}{l}\text { Nair et al. } \\
\text { (2011) }\end{array}$ & 14 & $\begin{array}{l}\text { Cortical 9, } \\
\text { subcortical } 5\end{array}$ & NA & $\begin{array}{l}\text { Moderate to } \\
\text { severe }\end{array}$ & 31 months & $\mathrm{FM}(30.1)$ & $\begin{array}{l}\text { Occupational } \\
\text { therapy }\end{array}$ & $\begin{array}{l}\text { Randomized, } \\
\text { parallel groups }\end{array}$ & ctDCS over cM1 & Five & 1 week & Pos \\
\hline $\begin{array}{l}\text { Tanaka et al. } \\
\text { (2011) }\end{array}$ & 8 & Subcortical & NA & $\begin{array}{l}\text { Mixed, lower } \\
\text { extremity }\end{array}$ & 21.1 months & $\begin{array}{l}\text { SIAS, knee } \\
\text { extension, GF }\end{array}$ & $\begin{array}{l}\text { Force knee } \\
\text { extension }\end{array}$ & $\begin{array}{l}\text { Randomized, } \\
\text { cross-over }\end{array}$ & atDCS over iM1 & One & NA & Pos \\
\hline $\begin{array}{l}\text { Stagg et al. } \\
\text { (2012) }\end{array}$ & 17 & $\begin{array}{l}\text { Cortical and } \\
\text { subcortical }\end{array}$ & $\begin{array}{l}\text { Ischemic 16, } \\
\text { hemorrhagic } 1\end{array}$ & Mixed & 37.9 months & $\begin{array}{l}\text { FM, GF, response } \\
\text { time task }\end{array}$ & NA & $\begin{array}{l}\text { Randomized, } \\
\text { double-blinded, } \\
\text { cross-over }\end{array}$ & $\begin{array}{l}\text { ctDCS over cM1 } \\
\text { and atDCS over } \\
\text { iM1 }\end{array}$ & One & NA & Pos \\
\hline $\begin{array}{l}\text { Zimerman } \\
\text { et al. (2012) }\end{array}$ & 12 & Subcortical & Ischemic & Mild & 30 months & MRC, FM, ASS, FT & SFTT & $\begin{array}{l}\text { Randomized, } \\
\text { double-blinded, } \\
\text { cross-over }\end{array}$ & ctDCS over cM1 & One & 3 months & Pos \\
\hline
\end{tabular}




\begin{tabular}{|c|c|c|c|c|c|c|c|c|c|c|c|c|}
\hline & $\begin{array}{c}\text { Number } \\
\text { of } \\
\text { patients }\end{array}$ & $\begin{array}{l}\text { Cortical/ } \\
\text { subcortical }\end{array}$ & $\begin{array}{c}\text { Ischemic/ } \\
\text { hemorrhagic }\end{array}$ & $\begin{array}{l}\text { Severity } \\
\text { of stroke }\end{array}$ & $\begin{array}{c}\text { Stroke } \\
\text { duration }\end{array}$ & $\begin{array}{c}\text { Motor } \\
\text { assessments } \\
\text { and outcomes }\end{array}$ & $\begin{array}{c}\text { Concomitant } \\
\text { therapy }\end{array}$ & $\begin{array}{l}\text { Study } \\
\text { design }\end{array}$ & $\begin{array}{c}\text { NIBS } \\
\text { Intervention }\end{array}$ & $\begin{array}{l}\text { Number } \\
\text { of } \\
\text { sessions }\end{array}$ & Follow-ups & Result \\
\hline $\begin{array}{l}\text { Danzl et al. } \\
\text { (2013) }\end{array}$ & 8 & NM & $\begin{array}{l}\text { Ischemic } 6 \text {, } \\
\text { hemorrhagic } 2\end{array}$ & Moderate & $\begin{array}{l}1.1- \\
11.6 \text { years }\end{array}$ & $\begin{array}{l}10 \mathrm{MWT}, \mathrm{TUG} \\
\text { BBS, FAC, SIS }\end{array}$ & $\begin{array}{l}\text { RGO-locomotor } \\
\text { training }\end{array}$ & $\begin{array}{l}\text { Randomized, } \\
\text { double-blind, } \\
\text { sham-controlled }\end{array}$ & atDCS over iM1 & 12 & 1 month & Pos \\
\hline $\begin{array}{l}\text { Giacobbe } \\
\text { et al. (2013) }\end{array}$ & 12 & NM & NM & Moderate & $>6$ months & MRC & $\begin{array}{l}\text { Robotic motor } \\
\text { practice }\end{array}$ & $\begin{array}{l}\text { Block- } \\
\text { randomized, } \\
\text { sham-controlled }\end{array}$ & atDCS over iM1 & Four & NA & Mix \\
\hline $\begin{array}{l}\text { Khedr et al. } \\
\text { (2013) }\end{array}$ & 40 & $\begin{array}{l}\text { Cortical 18, } \\
\text { cortico- } \\
\text { subcortical } 8 \text {, } \\
\text { subcortical } 14\end{array}$ & Ischemic & Moderate & $\begin{array}{l}\text { Anodal } \\
13.8 \pm 5.8 \\
\text { cathodal } \\
12.3 \pm 4.4 \\
\text { sham } \\
12.6 \pm \\
4.6 \text { days }\end{array}$ & $\begin{array}{l}\text { NIHSS, OMCASS, } \\
\text { BI, MRC }\end{array}$ & $\begin{array}{l}\text { Occupational } \\
\text { therapy within } \\
1 \mathrm{~h} \text { after } \\
\text { stimulation }\end{array}$ & $\begin{array}{l}\text { Single-center, } \\
\text { randomized, } \\
\text { double-blind, } \\
\text { sham-controlled }\end{array}$ & $\begin{array}{l}\text { atDCS over iM1, } \\
\text { ctDCS over cM1 }\end{array}$ & 6 days & $1-3$ months & Pos \\
\hline $\begin{array}{l}\text { Lefebvre } \\
\text { et al. (2012) }\end{array}$ & 18 & $\begin{array}{l}\text { Cortical 11, } \\
\text { subcortical } 7\end{array}$ & $\begin{array}{l}\text { Ischemic 16, } \\
\text { hemorrhagic } 2\end{array}$ & Moderate & $\begin{array}{l}2.6 \pm \\
1.5 \text { years }\end{array}$ & PP, GF & $\begin{array}{l}\text { Visuomotor } \\
\text { learning task }\end{array}$ & $\begin{array}{l}\text { Randomized, } \\
\text { cross-over, } \\
\text { sham- } \\
\text { controlled, } \\
\text { double-blind }\end{array}$ & Dual-tDCS & One & 1 week & Pos \\
\hline $\begin{array}{l}\text { Rossi et al. } \\
\text { (2013) }\end{array}$ & 50 & $\begin{array}{l}\text { Cortical 3, } \\
\text { cortico- } \\
\text { subcortical 35, } \\
\text { subcortical } 12\end{array}$ & Ischemic & $\begin{array}{l}\text { Moderate to } \\
\text { severe }\end{array}$ & 2 days & FM, NIHSS & NA & $\begin{array}{l}\text { Single-center, } \\
\text { randomized, } \\
\text { double-blind, } \\
\text { sham-controlled }\end{array}$ & atDCS over iM1 & Five & $\begin{array}{l}5 \text { days, } \\
3 \text { months }\end{array}$ & Neg \\
\hline $\begin{array}{l}\text { Sohn et al. } \\
\text { (2013) }\end{array}$ & 11 & Subcortical & $\begin{array}{l}\text { Ischemic } 4 \text {, } \\
\text { hemorrhagic } 7\end{array}$ & Severe & $\begin{array}{l}63.00 \pm \\
17.27 \text { days }\end{array}$ & $\begin{array}{l}\text { Static postural } \\
\text { stability, isometric } \\
\text { strength }\end{array}$ & NA & $\begin{array}{l}\text { Randomized, } \\
\text { cross-over, } \\
\text { sham-controlled }\end{array}$ & atDCS over iM1 & Two & NA & Mix \\
\hline $\begin{array}{l}\text { Au-Yeung } \\
\text { et al. (2014) }\end{array}$ & 10 & NM & $\begin{array}{l}\text { Ischemic } 8 \text {, } \\
\text { hemorrhagic } 2\end{array}$ & $\begin{array}{l}\text { Mild to } \\
\text { moderate }\end{array}$ & $\begin{array}{l}8.3 \pm \\
3.2 \text { years }\end{array}$ & PP, Stroop test & NA & $\begin{array}{l}\text { Double-blind, } \\
\text { sham- } \\
\text { controlled, } \\
\text { randomized, } \\
\text { cross-over }\end{array}$ & $\begin{array}{l}\text { atDCS over iM1, } \\
\text { ctDCS over cM1 }\end{array}$ & Three & NA & Mix \\
\hline $\begin{array}{l}\text { Fusco et al. } \\
\text { (2014) }\end{array}$ & 11 & NM & Ischemic & Mixed & $<30$ days & $\begin{array}{l}\text { CNS, BI, 9HPT, } \\
\text { FM, TUG, 10MWT, } \\
6 M W T, \text { RMI, FAC }\end{array}$ & $\begin{array}{l}\text { Rehabilitative } \\
\text { training }\end{array}$ & $\begin{array}{l}\text { Double-blind, } \\
\text { randomized, } \\
\text { sham-controlled }\end{array}$ & ctDCS over cM1 & 10 & $\begin{array}{l}30 \\
75-110 \text { days }\end{array}$ & Neg \\
\hline $\begin{array}{l}\text { Lefebvre } \\
\text { et al. (2014) }\end{array}$ & 19 & $\begin{array}{l}\text { Cortical 8, } \\
\text { subcortical } 11\end{array}$ & $\begin{array}{l}\text { Ischemic } 17 \\
\text { hemorrhagic } 2\end{array}$ & Moderate & $4 \pm 2$ years & PP, PG & NA & $\begin{array}{l}\text { Randomized, } \\
\text { cross-over, } \\
\text { sham- } \\
\text { controlled, } \\
\text { double-blind }\end{array}$ & Dual-tDCS & One & NA & Pos \\
\hline $\begin{array}{l}\text { O'Shea et al. } \\
\text { (2014) }\end{array}$ & 13 & $\begin{array}{l}\text { Cortical 6, } \\
\text { subcortical } 7\end{array}$ & $\begin{array}{l}\text { Ischemic } 12 \text {, } \\
\text { hemorrhagic } 1\end{array}$ & Moderate & $1.5-5.8$ years & sRT, FM, WMFT & NA & $\begin{array}{l}\text { Cross-over, } \\
\text { sham- } \\
\text { controlled, } \\
\text { single-blind }\end{array}$ & $\begin{array}{l}\text { atDCS over iM1, } \\
\text { ctDCS over } \\
\text { cM1, Dual-tDCS }\end{array}$ & One & NA & Mix \\
\hline
\end{tabular}




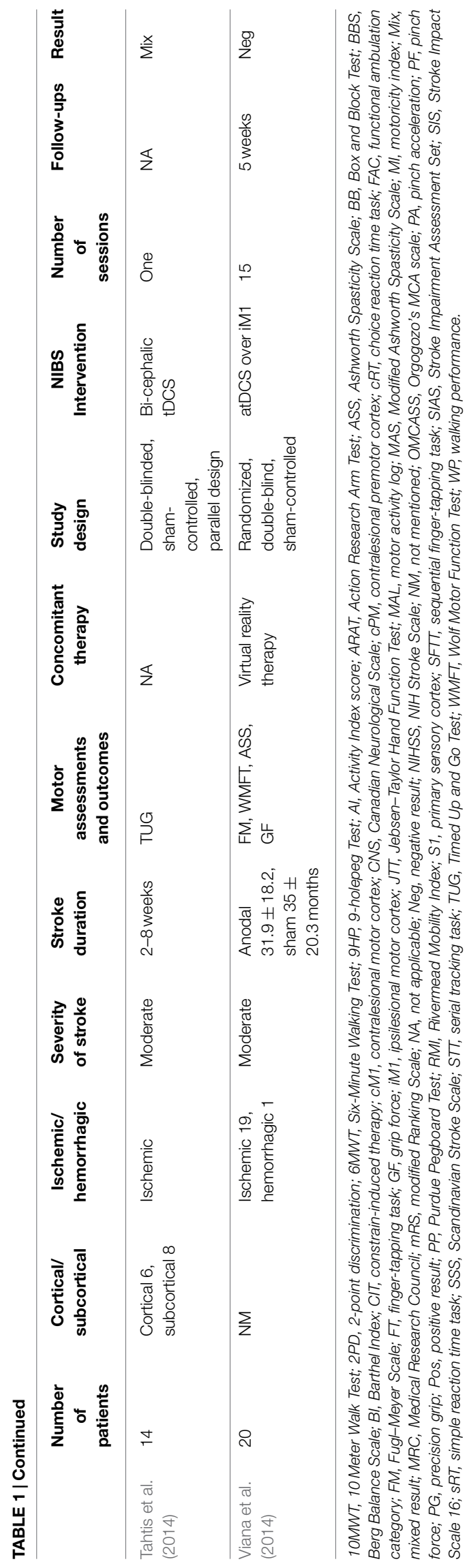

share in part similar mechanisms, it has been proposed that simultaneous application could be more or perhaps only effective (Hummel et al., 2008b; Bolognini et al., 2009). Stagg et al. (2011) systematically addressed this issue focusing on tDCS. In their study, anodal tDCS applied during an explicit sequence-learning task was associated with faster learning, whereas cathodal tDCS and anodal tDCS applied prior to the learning task was associated with slower learning (Stagg et al., 2011). This concept might hold true for stroke patients. In a recent single-center randomized, double-blind, sham-controlled study anodal tDCS applied to iM1 without conjunctive motor training could not elicit significant differences in Fugl-Meyer motor scores in-between stimulation groups in 50 acute stroke patients (Rossi et al., 2013). In this regard, it can be speculated that only concomitant combination of motor training with NIBS leads to additive or even supra-additive longer-lasting effects.

A remaining crucial question is in which state, acute, subacute, or chronic phase of the recovery process NIBS should be applied. To date, this issue cannot be answered sufficiently, but some theoretical consideration can be drawn. A benefit of early stimulation could be the enhanced adaptive plasticity in the acute and sub-acute phase (Ward, 2004; Nudo, 2006; Hummel et al., 2008b). Whereas, an advantage of late stimulation could be the lower risk of interfering with rescue of critically nourished neurons and of inducing neuronal toxicity after glial scar formation in the chronic phase (Hummel et al., 2008b). Moreover, when chronic patients show a more stable deficit, it is easier to evaluate possible behavioral effects of NIBS protocols (Hummel et al., 2008b). To conclude, more systematic studies are needed to find the optimal time point for application of a plasticity-inducing protocols.

\section{How to Stimulate?}

For methodological reasons, rTMS protocols, where subjects have to sit still during the intervention and cannot perform intensive motor training, are usually applied in an offline approach, utilizing the induced after-effects in the order of 30-60 min (Ziemann et al., 2008).

To increase effectivity, a promising complementary strategy could be the application of NIBS in a multi-session design. The magnitude of motor improvement in chronic stroke patients was increased over time by repetitive daily rTMS sessions (Fregni et al., 2006). In a complementary study, daily cathodal tDCS sessions to cM1 resulted in an augmented motor improvement when compared to a single-session design. Interestingly, this additive effect was not apparent in a design with weekly sessions (Boggio et al., 2007). An alternative compelling approach could be the use of spaced stimulation patterns, with multiple daily sessions. This might lead to prolonged after-effects via late-phase LTP/LTD-like neuroplasticity (Goldsworthy et al., 2014).

Lindenberg et al. (2010) have tested the effect of multifocal stimulation. In their study, they paired bihemispheric tDCS (anodal to iM1 and cathodal tDCS to cM1) with simultaneous occupational therapy (Lindenberg et al., 2010). Bihemispheric real stimulation resulted in a significant greater improvement in motor function when compared to sham stimulation. Although, 


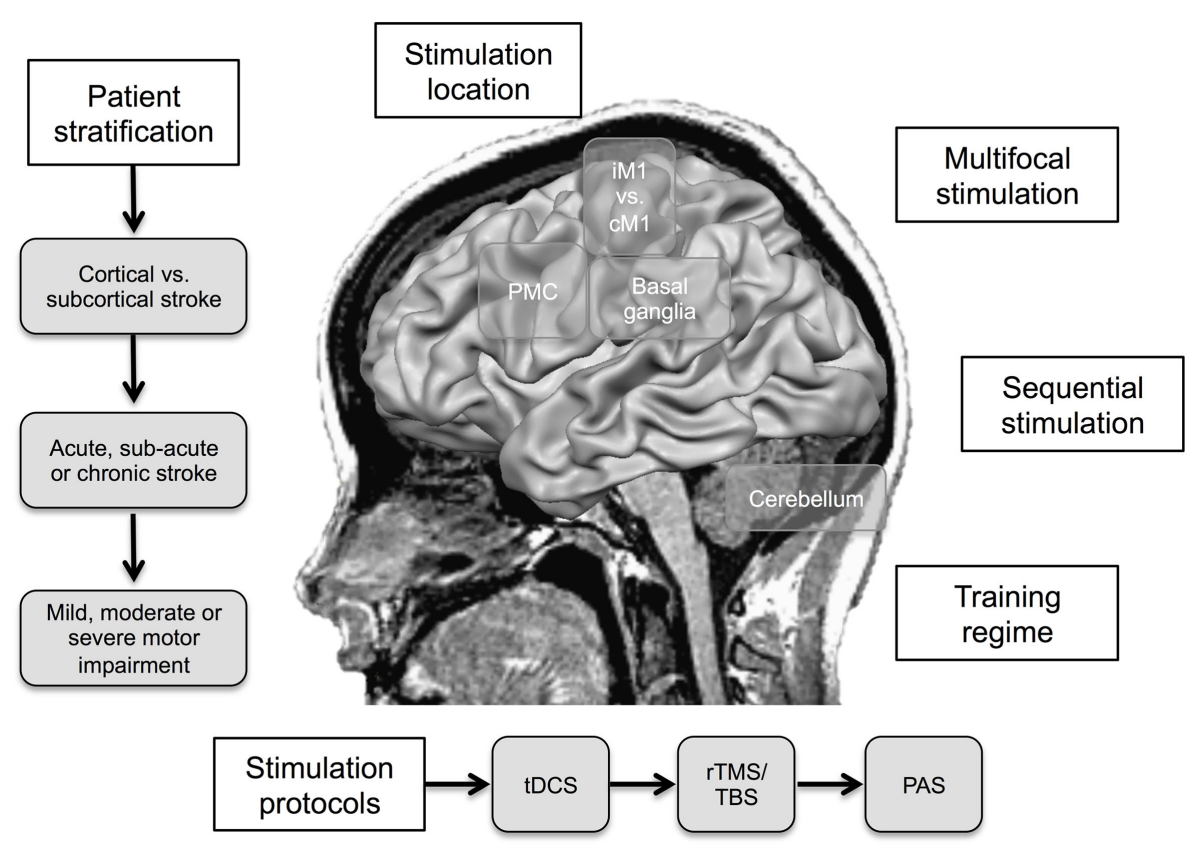

FIGURE 2 | Proposed strategies to increase the effect of non-invasive brain stimulation (NIBS) in motor recovery after stroke. iM1, ipsilesional primary motor cortex; CM1, contralesional primary motor cortex; PMC, premotor cortex; tDCS, transcranial direct current stimulation; rTMS, repetitive transcranial magnetic stimulation; TBS, theta-burst stimulation; PAS, paired associative stimulation.

TABLE 2 | Pros and cons of potential strategies for increasing the effect of non-invasive brain stimulation.

\begin{tabular}{|c|c|c|}
\hline Strategy & Pros & Cons \\
\hline Stimulation of iM1 & $\begin{array}{l}\text { - Direct enhancement of the reduced participation in the } \\
\text { incompletely recovered motor network after stroke }\end{array}$ & $\begin{array}{l}\text { - Higher risk of adverse effects due to induction of excitotoxicity } \\
\text { in the penumbra and shunting of electrical current }\end{array}$ \\
\hline Stimulation of $\mathrm{cM} 1$ & - Stimulation of intact cortical areas & - Inhibitory stimulation might also impair complex motor function \\
\hline $\begin{array}{l}\text { Stimulation of secondary } \\
\text { sensorimotor areas }\end{array}$ & $\begin{array}{l}\text { - Stimulation of intact cortical areas } \\
\text { - Modulation of cortico-cortical connections to M1 }\end{array}$ & - More difficult to target \\
\hline $\begin{array}{l}\text { Stimulation of the } \\
\text { cerebellum }\end{array}$ & $\begin{array}{l}\text { - Stimulation of intact cortical areas } \\
\text { - Alternative target within the motor learning network }\end{array}$ & $\begin{array}{l}\text { - More difficult to target } \\
\text { - Comparable high discomfort of cerebellar rTMS protocols }\end{array}$ \\
\hline $\begin{array}{l}\text { Simultaneous application of } \\
\text { a motor training paradigm }\end{array}$ & - Simultaneous modulation of LTP-/LTD-like mechanisms & $\begin{array}{l}\text { - Unfavorable homeostatic interactions } \\
\text { - Not feasible for most rTMS protocols }\end{array}$ \\
\hline $\begin{array}{l}\text { Stimulation in the acute or } \\
\text { sub-acute phase }\end{array}$ & - Enhanced adaptive plasticity & - Higher risk of adverse effects \\
\hline $\begin{array}{l}\text { Stimulation in the chronic } \\
\text { phase }\end{array}$ & $\begin{array}{l}\text { - More stable deficit } \\
\text { - Lower risk of adverse effects }\end{array}$ & - Reestablished growth/plasticity inhibition \\
\hline Multi-session stimulation & $\begin{array}{l}\text { - Enhancement of plasticity, e.g., induction of late-phase } \\
\text { LTP/LTD-like neuroplasticity }\end{array}$ & - More complex and time-consuming \\
\hline Multifocal stimulation & $\begin{array}{l}\text { - Modulation of multiple nodes of the motor network } \\
\text { - Induction of additive or supra-additive effects }\end{array}$ & - Higher risk of adverse effects, e.g., shunting of current \\
\hline Sequential stimulation & $\begin{array}{l}\text { - Time-dependent modulation of multiple nodes of the motor } \\
\text { network }\end{array}$ & - More complex setup \\
\hline Patterned rTMS protocols & $\begin{array}{l}\text { - Shorter delivery time } \\
\text { - Proposed potent modulatory aftereffect }\end{array}$ & $\begin{array}{l}\text { - Higher risk of adverse effects } \\
\text { - Need of a more complex and expensive setup } \\
\text { - Mixed results }\end{array}$ \\
\hline
\end{tabular}

an additive effect in motor function scores has been proposed, it remains unclear whether the bilateral approach is superior to a unilateral due to lack of sufficient control conditions. Sequential stimulation of different areas involved during the motor learning process could be an alternative strategy (Grimaldi et al., 2014).
Finally, a promising new interventional approach is the use of patterned rTMS protocols, like TBS. Proposed advantages are shorter delivery time and potent modulatory after-effects (Edwardson et al., 2013). In a first small proof-of-principle study, iTBS applied to iM1 improved motor function in chronic stroke 
patients (Talelli et al., 2007). In a larger semi-randomized clinical trial, in which iTBS to iM1 was combined with physical therapy over 10 days, this adjuvant effect of iTBS could not be replicated (Talelli et al., 2012).

Lastly, we want to point out that despite its similarities, rTMS, tDCS, and PAS differ in spatial and temporal resolution and the underlying neurophysiological mechanisms (Gandiga et al., 2006; Ziemann et al., 2008).

\section{Enhancement of Motor Learning by Innovative Training Regimes}

In addition to adjuvant NIBS, stroke patients could potentially benefit from the use of innovative training regimes.

In a standard neurorehabilitative training session, usually a variety of different skills are practiced. The consolidation of the learned skills is highly dependent on the practice protocols. Performance during training is typically better, when the different skills are trained in a sequential order - block design. A random, intermingled training usually results in greater offline learning. This effect is called contextual interference (Battig, 1972). Recent fMRI studies provided evidence that random practice results in greater neuronal activity in regions crucial for preparation and production of learned motor skills at the end of training relative to block practice. It has been suggested that this persistent active preparation benefits offline learning (Wymbs and Grafton, 2009). Schweighofer et al. (2011) investigated the contextual interference effect in sub-acute stroke patients. In their study, patients with normal visuospatial working memory showed less long-term forgetting in a visuomotor task in the random training condition. Whereas, patients with low visuospatial working memory exhibited little long-term forgetting in both conditions (Schweighofer et al., 2011).

It has been suggested that the feedback given on a training session modulates its memory formation. This has been extensively investigated in animal models using associative learning paradigms. It was shown that reward of good performance and punishment of bad performance can modulate its memory consolidation (Tempel et al., 1983). Abe et al. (2011) recently studied this phenomenon in human motor skill learning in young healthy subjects. Learning under reward conditions enhanced long-term retention via significant offline memory gains. Whereas, training under neutral and punished conditions exhibited offline memory losses (Abe et al., 2011). Proposed neuroanatomical substrates of reward processing are dopaminergic neurotransmission (Zald et al., 2004) and in interconnected network involving the orbitofrontal cortex, amygdala, and ventral striatum (O'Doherty, 2004). Interestingly, a recent study provided evidence that high reward can facilitate learning of an ankle robotics motor training task in chronic stroke patients (Goodman et al., 2014).

\section{Future Directions}

Non-invasive brain stimulation techniques are a promising adjuvant strategy for enhancing post-stroke recovery. However, many open questions remain. Future studies have to investigate possible mechanisms, the optimal site, time point and type of stimulation (Hummel et al., 2008b). Since stroke is a heterogeneous condition, it is very likely that patients will benefit most from individually tailored stimulation protocols. This could be achieved by patient stratification in regards to the clinical deficit, lesion location, lesion size, comorbidities, time in the recovery process, age, and gender (Hummel et al., 2008b). Also standardized algorithms, e.g., the PREP algorithm recently proposed by Stinear et al. (2012), could be useful for patient stratification. Another promising strategy could be the combination of different stimulation techniques. For instance, the combination of PNS and tDCS to iM1 in chronic stroke patients resulted in superior performance in a sequential motor task compared to unimodal stimulation or training alone (Celnik et al., 2009).

Overall, there is a great need for the development and testing of novel innovative interventional strategies individually tailored to the patients' prerequisites. To achieve these goals, mechanism driven proof-of principle studies and large, multicenter, placebocontrolled trials are still necessary. Furthermore, a close cooperation between basic researchers and clinicians is needed to develop the field and bring innovative ideas from bench to bedside into daily clinical life.

\section{Limitations of Non-Invasive Brain Stimulation}

In spite of the encouraging perspective of NIBS, limitations and unresolved issues remain.

A recent study by Wiethoff and colleagues emphasized the high in-between subject variability of tDCS protocols. They applied 10 min of tDCS over M1 in 53 healthy subjects in a crossover design and assed the corticospinal excitability by measuring the MEP amplitude. At group level, anodal tDCS facilitated the MEP whereas cathodal tDCS showed no significant effect. In inspection of the single subject data, only $36 \%$ showed the expected facilitatory anodal and inhibitory cathodal effect, $21 \%$ showed a reversed pattern, $38 \%$ showed a polarity independent facilitatory, and 5\% an inhibitory effect (Wiethoff et al., 2014). In this regard, several factors, which influence the response to plasticity-inducing NIBS protocols, like the history of synaptic activity, genetic polymorphisms of neurotrophins, use of CNSactive drugs, attention, age, gender, circadian rhythms, aerobic exercise, among others have been identified (Ridding and Ziemann, 2010).

It is of note that NIBS can potentially induce adverse effects, like headache (occasionally), skin burning (rare), seizures (very rare), and eyelid myokymia (very rare) (Brunoni et al., 2011; Wessel et al., 2013).

\section{Acknowledgments}

This research was supported by the German Research Foundation SFB 936-C4 to FCH. 


\section{References}

Abe, M., Schambra, H., Wassermann, E. M., Luckenbaugh, D., Schweighofer, N., and Cohen, L. G. (2011). Reward improves long-term retention of a motor memory through induction of offline memory gains. Curr. Biol. 21, 557-562. doi:10.1016/j.cub.2011.02.030

Ameli, M., Grefkes, C., Kemper, F., Riegg, F. P., Rehme, A. K., Karbe, H., et al. (2009). Differential effects of high-frequency repetitive transcranial magnetic stimulation over ipsilesional primary motor cortex in cortical and subcortical middle cerebral artery stroke. Ann. Neurol. 66, 298-309. doi:10.1002/ana.21725

Antal, A., and Paulus, W. (2013). Transcranial alternating current stimulation (tACS). Front. Hum. Neurosci. 7:317. doi:10.3389/fnhum.2013.00317

Au-Yeung, S. S., Wang, J., Chen, Y., and Chua, E. (2014). Transcranial direct current stimulation to primary motor area improves hand dexterity and selective attention in chronic stroke. Am. J. Phys. Med. Rehabil. 93, 1057-1064. doi:10. 1097/PHM.0000000000000127

Avenanti, A., Coccia, M., Ladavas, E., Provinciali, L., and Ceravolo, M. G. (2012). Low-frequency rTMS promotes use-dependent motor plasticity in chronic stroke: a randomized trial. Neurology 78, 256-264. doi:10.1212/WNL. 0b013e3182436558

Battig, W. (1972). Intratask Interference as a Source of Facilitation in Transfer and Retention. New York, NY: Academic Press.

Baztan, J. J., Perez-Martinez, D. A., Fernandez-Alonso, M., Aguado-Ortego, R., Bellando-Alvarez, G., and de la Fuente-Gonzalez, A. M. (2007). [Prognostic factors of functional recovery in very elderly stroke patients: a one-year follow-up study]. Rev. Neurol. 44, 577-583.

Boggio, P. S., Castro, L. O., Savagim, E. A., Braite, R., Cruz, V. C., Rocha, R. R., et al. (2006). Enhancement of non-dominant hand motor function by anodal transcranial direct current stimulation. Neurosci. Lett. 404, 232-236. doi:10. 1016/j.neulet.2006.05.051

Boggio, P. S., Nunes, A., Rigonatti, S. P., Nitsche, M. A., Pascual-Leone, A., and Fregni, F. (2007). Repeated sessions of noninvasive brain DC stimulation is associated with motor function improvement in stroke patients. Restor. Neurol. Neurosci. 25, 123-129.

Bolognini, N., Pascual-Leone, A., and Fregni, F. (2009). Using non-invasive brain stimulation to augment motor training-induced plasticity. J. Neuroeng. Rehabil. 6, 8. doi:10.1186/1743-0003-6-8

Bolognini, N., Vallar, G., Casati, C., Latif, L. A., El-Nazer, R., Williams, J., et al. (2011). Neurophysiological and behavioral effects of tDCS combined with constraint-induced movement therapy in poststroke patients. Neurorehabil. Neural Repair 25, 819-829. doi:10.1177/1545968311411056

Bortoletto, M., Pellicciari, M. C., Rodella, C., and Miniussi, C. (2014). The interaction with task-induced activity is more important than polarization: a tDCS study. Brain Stimulat. 8, 269-276. doi:10.1016/j.brs.2014.11.006

Bradnam, L. V., Stinear, C. M., Barber, P. A., and Byblow, W. D. (2011). Contralesional hemisphere control of the proximal paretic upper limb following stroke. Cereb. Cortex 22, 2662-2671. doi:10.1093/cercor/bhr344

Brodie, S. M., Meehan, S., Borich, M. R., and Boyd, L. A. (2014). 5 Hz repetitive transcranial magnetic stimulation over the ipsilesional sensory cortex enhances motor learning after stroke. Front. Hum. Neurosci. 8:143. doi:10.3389/fnhum. 2014.00143

Brown, C. E., Boyd, J. D., and Murphy, T. H. (2010). Longitudinal in vivo imaging reveals balanced and branch-specific remodeling of mature cortical pyramidal dendritic arbors after stroke. J. Cereb. Blood Flow Metab. 30, 783-791. doi:10. 1038/jcbfm.2009.241

Brunoni, A. R., Amadera, J., Berbel, B., Volz, M. S., Rizzerio, B. G., and Fregni, F. (2011). A systematic review on reporting and assessment of adverse effects associated with transcranial direct current stimulation. Int. J. Neuropsychopharmacol. 14, 1133-1145. doi:10.1017/S1461145710001690

Carmichael, S. T., Wei, L., Rovainen, C. M., and Woolsey, T. A. (2001). New patterns of intracortical projections after focal cortical stroke. Neurobiol. Dis. 8, 910-922. doi:10.1006/nbdi.2001.0425

Castel-Lacanal, E., Gerdelat-Mas, A., Marque, P., Loubinoux, I., and SimonettaMoreau, M. (2007). Induction of cortical plastic changes in wrist muscles by paired associative stimulation in healthy subjects and post-stroke patients. Exp. Brain Res. 180, 113-122. doi:10.1007/s00221-006-0844-5

Castel-Lacanal, E., Marque, P., Tardy, J., de Boissezon, X., Guiraud, V., Chollet, F., et al. (2009). Induction of cortical plastic changes in wrist muscles by paired associative stimulation in the recovery phase of stroke patients. Neurorehabil. Neural Repair 23, 366-372. doi:10.1177/1545968308322841

Celnik, P., Paik, N. J., Vandermeeren, Y., Dimyan, M., and Cohen, L. G. (2009). Effects of combined peripheral nerve stimulation and brain polarization on performance of a motor sequence task after chronic stroke. Stroke 40, 1764-1771. doi:10.1161/STROKEAHA.108.540500

Chang, W. H., Kim, Y. H., Bang, O. Y., Kim, S. T., Park, Y. H., and Lee, P. K. (2010). Long-term effects of rTMS on motor recovery in patients after subacute stroke. J. Rehabil. Med. 42, 758-764. doi:10.2340/16501977-0590

Chang, W. H., Kim, Y. H., Yoo, W. K., Goo, K. H., Park, C. H., Kim, S. T., et al. (2012). rTMS with motor training modulates cortico-basal gangliathalamocortical circuits in stroke patients. Restor. Neurol. Neurosci. 30, 179-189. doi:10.3233/RNN-2012-110162

Cicinelli, P., Pasqualetti, P., Zaccagnini, M., Traversa, R., Oliveri, M., and Rossini, P. M. (2003). Interhemispheric asymmetries of motor cortex excitability in the postacute stroke stage: a paired-pulse transcranial magnetic stimulation study. Stroke 34, 2653-2658. doi:10.1161/01.STR.0000092122.96722.72

Conforto, A. B., Anjos, S. M., Saposnik, G., Mello, E. A., Nagaya, E. M., Santos, W. Jr., et al. (2012). Transcranial magnetic stimulation in mild to severe hemiparesis early after stroke: a proof of principle and novel approach to improve motor function. J. Neurol. 259, 1399-1405. doi:10.1007/ s00415-011-6364-7

Dafotakis, M., Grefkes, C., Eickhoff, S. B., Karbe, H., Fink, G. R., and Nowak, D. A (2008). Effects of rTMS on grip force control following subcortical stroke. Exp. Neurol. 211, 407-412. doi:10.1016/j.expneurol.2008.02.018

Danzl, M. M., Chelette, K. C., Lee, K., Lykins, D., and Sawaki, L. (2013). Brain stimulation paired with novel locomotor training with robotic gait orthosis in chronic stroke: a feasibility study. NeuroRehabilitation 33, 67-76. doi:10.3233/ NRE-130929

Dayan, E., and Cohen, L. G. (2011). Neuroplasticity subserving motor skill learning. Neuron 72, 443-454. doi:10.1016/j.neuron.2011.10.008

Debas, K., Carrier, J., Orban, P., Barakat, M., Lungu, O., Vandewalle, G., et al. (2010). Brain plasticity related to the consolidation of motor sequence learning and motor adaptation. Proc. Natl. Acad. Sci. U.S.A. 107, 17839-17844. doi:10.1073/ pnas. 1013176107

Doyon, J., and Benali, H. (2005). Reorganization and plasticity in the adult brain during learning of motor skills. Curr. Opin. Neurobiol. 15, 161-167. doi:10.1016/ j.conb.2005.03.004

Doyon, J., Penhune, V., and Ungerleider, L. G. (2003). Distinct contribution of the cortico-striatal and cortico-cerebellar systems to motor skill learning. Neuropsychologia 41, 252-262. doi:10.1016/S0028-3932(02)00158-6

Edwardson, M. A., Lucas, T. H., Carey, J. R., and Fetz, E. E. (2013). New modalities of brain stimulation for stroke rehabilitation. Exp. Brain Res. 224, 335-358. doi:10.1007/s00221-012-3315-1

Emara, T. H., Moustafa, R. R., Elnahas, N. M., Elganzoury, A. M., Abdo, T. A. Mohamed, S. A., et al. (2010). Repetitive transcranial magnetic stimulation at $1 \mathrm{~Hz}$ and $5 \mathrm{~Hz}$ produces sustained improvement in motor function and disability after ischaemic stroke. Eur. J. Neurol. 17, 1203-1209. doi:10.1111/j.1468-1331. 2010.03000.x

Etoh, S., Noma, T., Ikeda, K., Jonoshita, Y., Ogata, A., Matsumoto, S., et al. (2013). Effects of repetitive trascranial magnetic stimulation on repetitive facilitation exercises of the hemiplegic hand in chronic stroke patients. J. Rehabil. Med. 45 843-847. doi:10.2340/16501977-1175

Floyer-Lea, A., and Matthews, P. M. (2005). Distinguishable brain activation networks for short- and long-term motor skill learning. J. Neurophysiol. 94, 512-518. doi:10.1152/jn.00717.2004

Fregni, F., Boggio, P. S., Mansur, C. G., Wagner, T., Ferreira, M. J., Lima, M. C., et al. (2005). Transcranial direct current stimulation of the unaffected hemisphere in stroke patients. Neuroreport 16, 1551-1555. doi:10.1097/01.wnr.0000177010. $44602.5 \mathrm{e}$

Fregni, F., Boggio, P. S., Valle, A. C., Rocha, R. R., Duarte, J., Ferreira, M. J., et al. (2006). A sham-controlled trial of a 5-day course of repetitive transcranial magnetic stimulation of the unaffected hemisphere in stroke patients. Stroke 37, 2115-2122. doi:10.1161/01.STR.0000231390.58967.6b

Fritsch, B., Reis, J., Martinowich, K., Schambra, H. M., Ji, Y., Cohen, L. G., et al. (2010). Direct current stimulation promotes BDNF-dependent synaptic plasticity: potential implications for motor learning. Neuron 66, 198-204. doi:10.1016/ j.neuron.2010.03.035 
Fusco, A., Assenza, F., Iosa, M., Izzo, S., Altavilla, R., Paolucci, S., et al. (2014). The ineffective role of cathodal tDCS in enhancing the functional motor outcomes in early phase of stroke rehabilitation: an experimental trial. Biomed Res. Int. 2014:547290. doi:10.1155/2014/547290

Galea, J. M., Vazquez, A., Pasricha, N., de Xivry, J. J., and Celnik, P. (2011). Dissociating the roles of the cerebellum and motor cortex during adaptive learning: the motor cortex retains what the cerebellum learns. Cereb. Cortex 21, 1761-1770. doi:10.1093/cercor/bhq246

Gandiga, P. C., Hummel, F. C., and Cohen, L. G. (2006). Transcranial DC stimulation (tDCS): a tool for double-blind sham-controlled clinical studies in brain stimulation. Clin. Neurophysiol. 117, 845-850. doi:10.1016/j.clinph.2005. 12.003

Gerloff, C., Bushara, K., Sailer, A., Wassermann, E. M., Chen, R., Matsuoka, T., et al. (2006). Multimodal imaging of brain reorganization in motor areas of the contralesional hemisphere of well recovered patients after capsular stroke. Brain 129(Pt 3), 791-808. doi:10.1093/brain/awh713

Giacobbe, V., Krebs, H. I., Volpe, B. T., Pascual-Leone, A., Rykman, A., Zeiarati, G., et al. (2013). Transcranial direct current stimulation (tDCS) and robotic practice in chronic stroke: the dimension of timing. NeuroRehabilitation 33, 49-56. doi:10.3233/NRE-130927

Goldsworthy, M. R., Pitcher, J. B., and Ridding, M. C. (2014). Spaced noninvasive brain stimulation: prospects for inducing long-lasting human cortical plasticity. Neurorehabil. Neural Repair doi:10.1177/1545968314562649

Goodman, R. N., Rietschel, J. C., Roy, A., Jung, B. C., Diaz, J., Macko, R. F., et al. (2014). Increased reward in ankle robotics training enhances motor control and cortical efficiency in stroke. J. Rehabil. Res. Dev. 51, 213-227. doi:10.1682/JRRD. 2013.02.0050

Greenough, W. T., Larson, J. R., and Withers, G. S. (1985). Effects of unilateral and bilateral training in a reaching task on dendritic branching of neurons in the rat motor-sensory forelimb cortex. Behav. Neural Biol. 44, 301-314. doi:10.1016/ S0163-1047(85)90310-3

Grefkes, C., Nowak, D. A., Eickhoff, S. B., Dafotakis, M., Kust, J., Karbe, H., et al. (2008). Cortical connectivity after subcortical stroke assessed with functional magnetic resonance imaging. Ann. Neurol. 63, 236-246. doi:10.1002/ana. 21228

Grefkes, C., Nowak, D. A., Wang, L. E., Dafotakis, M., Eickhoff, S. B., and Fink, G. R. (2010). Modulating cortical connectivity in stroke patients by rTMS assessed with fMRI and dynamic causal modeling. Neuroimage 50, 233-242. doi:10.1016/ j.neuroimage.2009.12.029

Grimaldi, G., Oulad Ben Taib, N., Manto, M., and Bodranghien, F. (2014). Marked reduction of cerebellar deficits in upper limbs following transcranial cerebellocerebral DC stimulation: tremor reduction and re-programming of the timing of antagonist commands. Front. Syst. Neurosci. 8:9. doi:10.3389/fnsys.2014.00009

Hallett, M. (2001). Plasticity of the human motor cortex and recovery from stroke. Brain Res. Brain Res. Rev. 36, 169-174. doi:10.1016/S0165-0173(01)00092-3

Hallett, M. (2007). Transcranial magnetic stimulation: a primer. Neuron 55, 187-199. doi:10.1016/j.neuron.2007.06.026

Halter, J. A., Carp, J. S., and Wolpaw, J. R. (1995). Operantly conditioned motoneuron plasticity: possible role of sodium channels. J. Neurophysiol. 73, 867-871.

Hamada, M., and Ugawa, Y. (2010). Quadripulse stimulation - a new patterned rTMS. Restor. Neurol. Neurosci. 28, 419-424. doi:10.3233/RNN-2010-0564

Heidenreich, P. A., Trogdon, J. G., Khavjou, O. A., Butler, J., Dracup, K., Ezekowitz, M. D., et al. (2011). Forecasting the future of cardiovascular disease in the United States: a policy statement from the American Heart Association. Circulation 123, 933-944. doi:10.1161/CIR.0b013e31820a55f5

Hesse, S., Waldner, A., Mehrholz, J., Tomelleri, C., Pohl, M., and Werner, C. (2011). Combined transcranial direct current stimulation and robot-assisted arm training in subacute stroke patients: an exploratory, randomized multicenter trial. Neurorehabil. Neural Repair 25, 838-846. doi:10.1177/1545968311413906

Hesse, S., Werner, C., Schonhardt, E. M., Bardeleben, A., Jenrich, W., and Kirker, S. G. (2007). Combined transcranial direct current stimulation and robot-assisted arm training in subacute stroke patients: a pilot study. Restor. Neurol. Neurosci. 25, 9-15.

Higgins, J., Koski, L., and Xie, H. (2013). Combining rTMS and task-oriented training in the rehabilitation of the arm after stroke: a pilot randomized controlled trial. Stroke Res. Treat. 2013, 539146. doi:10.1155/2013/539146

Hikosaka, O., Nakamura, K., Sakai, K., and Nakahara, H. (2002). Central mechanisms of motor skill learning. Curr. Opin. Neurobiol. 12, 217-222. doi:10.1016/ S0959-4388(02)00307-0
Hosp, J. A., and Luft, A. R. (2011). Cortical plasticity during motor learning and recovery after ischemic stroke. Neural Plast. 2011, 871296. doi:10.1155/2011/ 871296

Huang, Y. Z., Edwards, M. J., Rounis, E., Bhatia, K. P., and Rothwell, J. C. (2005). Theta burst stimulation of the human motor cortex. Neuron 45, 201-206. doi:10. 1016/j.neuron.2004.12.033

Hummel, F., Celnik, P., Giraux, P., Floel, A., Wu, W. H., Gerloff, C., et al. (2005). Effects of non-invasive cortical stimulation on skilled motor function in chronic stroke. Brain 128(Pt 3), 490-499. doi:10.1093/brain/awh369

Hummel, F. C., Celnik, P., Pascual-Leone, A., Fregni, F., Byblow, W. D., Buetefisch, C. M., et al. (2008a). Controversy: noninvasive and invasive cortical stimulation show efficacy in treating stroke patients. Brain Stimul. 1, 370-382. doi:10.1016/ j.brs.2008.09.003

Hummel, F. C., Celnik, P., Pascual-Leone, A., Fregni, F., Byblow, W. D., Buetefisch, C. M., et al. (2008b). Controversy: noninvasive and invasive cortical stimulation show efficacy in treating stroke patients. Brain Stimulat. 1, 370-382. doi:10.1016/ j.brs.2008.09.003

Hummel, F. C., and Cohen, L. G. (2005). Drivers of brain plasticity. Curr. Opin. Neurol. 18, 667-674. doi:10.1097/01.wco.0000189876.37475.42

Hummel, F. C., and Cohen, L. G. (2006). Non-invasive brain stimulation: a new strategy to improve neurorehabilitation after stroke? Lancet Neurol. 5, 708-712. doi:10.1016/S1474-4422(06)70525-7

Hummel, F. C., Voller, B., Celnik, P., Floel, A., Giraux, P., Gerloff, C., et al. (2006) Effects of brain polarization on reaction times and pinch force in chronic stroke. BMC Neurosci. 7:73. doi:10.1186/1471-2202-7-73

Johansen-Berg, H., Dawes, H., Guy, C., Smith, S. M., Wade, D. T., and Matthews, P. M. (2002). Correlation between motor improvements and altered fMRI activity after rehabilitative therapy. Brain 125(Pt 12), 2731-2742. doi:10.1093/brain/ awf 282

Jorgensen, H. S. (1996). The Copenhagen stroke study experience. J. Stroke Cerebrovasc. Dis. 6, 5-16. doi:10.1016/S1052-3057(96)80020-6

Jorgensen, H. S., Nakayama, H., Raaschou, H. O., and Olsen, T. S. (1999). Stroke. neurologic and functional recovery the Copenhagen stroke study. Phys. Med. Rehabil. Clin. N. Am. 10, 887-906.

Khedr, E. M., Abdel-Fadeil, M. R., Farghali, A., and Qaid, M. (2009). Role of 1 and $3 \mathrm{~Hz}$ repetitive transcranial magnetic stimulation on motor function recovery after acute ischaemic stroke. Eur. J. Neurol. 16, 1323-1330. doi:10.1111/ j.1468-1331.2009.02746.x

Khedr, E. M., Ahmed, M. A., Fathy, N., and Rothwell, J. C. (2005). Therapeutic trial of repetitive transcranial magnetic stimulation after acute ischemic stroke. Neurology 65, 466-468. doi:10.1212/01.wnl.0000173067.84247.36

Khedr, E. M., Shawky, O. A., El-Hammady, D. H., Rothwell, J. C., Darwish, E. S., Mostafa, O. M., et al. (2013). Effect of anodal versus cathodal transcranial direct current stimulation on stroke rehabilitation: a pilot randomized controlled trial. Neurorehabil. Neural Repair 27, 592-601. doi:10.1177/1545968313484808

Kim, D. Y., Lim, J. Y., Kang, E. K., You, D. S., Oh, M. K., Oh, B. M., et al. (2010). Effect of transcranial direct current stimulation on motor recovery in patients with subacute stroke. Am. J. Phys. Med. Rehabil. 89, 879-886. doi:10.1097/PHM. 0b013e3181f70aa7

Kim, Y. H., You, S. H., Ko, M. H., Park, J. W., Lee, K. H., Jang, S. H., et al. (2006) Repetitive transcranial magnetic stimulation-induced corticomotor excitability and associated motor skill acquisition in chronic stroke. Stroke 37, 1471-1476 doi:10.1161/01.STR.0000221233.55497.51

Kim, Y. J., Ku, J., Cho, S., Kim, H. J., Cho, Y. K., Lim, T., et al. (2014a). Facilitation of corticospinal excitability by virtual reality exercise following anodal transcranial direct current stimulation in healthy volunteers and subacute stroke subjects. $J$. Neuroeng. Rehabil. 11, 124. doi:10.1186/1743-0003-11-124

Kim, W. S., Jung, S. H., Oh, M. K., Min, Y. S., Lim, J. Y., and Paik, N. J. (2014b). Effect of repetitive transcranial magnetic stimulation over the cerebellum on patients with ataxia after posterior circulation stroke: a pilot study. J. Rehabil. Med. 46, 418-423. doi:10.2340/16501977-1802

Kochanek, K. D., Kirmeyer, S. E., Martin, J. A., Strobino, D. M., and Guyer, B. (2012). Annual summary of vital statistics: 2009. Pediatrics 129, 338-348. doi:10. 1542/peds.2011-3435

Krakauer, J. W. (2006). Motor learning: its relevance to stroke recovery and neurorehabilitation. Curr. Opin. Neurol. 19, 84-90. doi:10.1097/01.wco.0000200544 29915.cc

Landi, S. M., Baguear, F., and Della-Maggiore, V. (2011). One week of motor adaptation induces structural changes in primary motor cortex that predict 
long-term memory one year later. J. Neurosci. 31, 11808-11813. doi:10.1523/ JNEUROSCI.2253-11.2011

Langhorne, P., Williams, B. O., Gilchrist, W., and Howie, K. (1993). Do stroke units save lives? Lancet 342, 395-398. doi:10.1016/0140-6736(93)92813-9

Lefebvre, S., Laloux, P., Peeters, A., Desfontaines, P., Jamart, J., and Vandermeeren, Y. (2012). Dual-tDCS enhances online motor skill learning and long-term retention in chronic stroke patients. Front. Hum. Neurosci. 6:343. doi:10.3389/ fnhum.2012.00343

Lefebvre, S., Thonnard, J. L., Laloux, P., Peeters, A., Jamart, J., and Vandermeeren, Y. (2014). Single session of dual-tDCS transiently improves precision grip and dexterity of the paretic hand after stroke. Neurorehabil. Neural Repair 28, 100-110. doi:10.1177/1545968313478485

Liepert, J., Zittel, S., and Weiller, C. (2007). Improvement of dexterity by single session low-frequency repetitive transcranial magnetic stimulation over the contralesional motor cortex in acute stroke: a double-blind placebo-controlled crossover trial. Restor. Neurol. Neurosci. 25, 461-465.

Lindenberg, R., Renga, V., Zhu, L. L., Nair, D., and Schlaug, G. (2010). Bihemispheric brain stimulation facilitates motor recovery in chronic stroke patients. Neurology 75, 2176-2184. doi:10.1212/WNL.0b013e318202013a

Lotze, M., Markert, J., Sauseng, P., Hoppe, J., Plewnia, C., and Gerloff, C. (2006). The role of multiple contralesional motor areas for complex hand movements after internal capsular lesion. J. Neurosci. 26, 6096-6102. doi:10.1523/JNEUROSCI. 4564-05.2006

Luft, A. R., Buitrago, M. M., Ringer, T., Dichgans, J., and Schulz, J. B. (2004). Motor skill learning depends on protein synthesis in motor cortex after training. J. Neurosci. 24, 6515-6520. doi:10.1523/JNEUROSCI.1034-04.2004

Madhavan, S., Weber, K. A. II, and Stinear, J. W. (2011). Non-invasive brain stimulation enhances fine motor control of the hemiparetic ankle: implications for rehabilitation. Exp. Brain Res. 209, 9-17. doi:10.1007/s00221-010-2511-0

Malcolm, M. P., Triggs, W. J., Light, K. E., Gonzalez Rothi, L. J., Wu, S., Reid, K., et al. (2007). Repetitive transcranial magnetic stimulation as an adjunct to constraintinduced therapy: an exploratory randomized controlled trial. Am. J. Phys. Med. Rehabil. 86, 707-715. doi:10.1097/PHM.0b013e31813e0de0

Mally, J., and Dinya, E. (2008). Recovery of motor disability and spasticity in poststroke after repetitive transcranial magnetic stimulation (rTMS). Brain Res. Bull. 76, 388-395. doi:10.1016/j.brainresbull.2007.11.019

Mansur, C. G., Fregni, F., Boggio, P. S., Riberto, M., Gallucci-Neto, J., Santos, C. M., et al. (2005). A sham stimulation-controlled trial of rTMS of the unaffected hemisphere in stroke patients. Neurology 64, 1802-1804. doi:10.1212/01.WNL. 0000161839.38079.92

Moos, K., Vossel, S., Weidner, R., Sparing, R., and Fink, G. R. (2012). Modulation of top-down control of visual attention by cathodal tDCS over right IPS. J. Neurosci. 32, 16360-16368. doi:10.1523/JNEUROSCI.6233-11.2012

Motamed Vaziri, P., Bahrpeyma, F., Firoozabadi, M., Forough, B., Hatef, B., Sheikhhoseini, R., et al. (2014). Low frequency repetitive transcranial magnetic stimulation to improve motor function and grip force of upper limbs of patients with hemiplegia. Iran. Red Crescent Med. J. 16, e13579. doi:10.5812/ircmj.13579

Muellbacher, W., Ziemann, U., Wissel, J., Dang, N., Kofler, M., Facchini, S., et al. (2002). Early consolidation in human primary motor cortex. Nature 415, 640-644. doi:10.1038/nature712

Murase, N., Duque, J., Mazzocchio, R., and Cohen, L. G. (2004). Influence of interhemispheric interactions on motor function in chronic stroke. Ann. Neurol. 55, 400-409. doi:10.1002/ana.10848

Nair, D. G., Renga, V., Lindenberg, R., Zhu, L., and Schlaug, G. (2011). Optimizing recovery potential through simultaneous occupational therapy and non-invasive brain-stimulation using tDCS. Restor. Neurol. Neurosci. 29, 411-420. doi:10. 3233/RNN-2011-0612

Nakayama, H., Jorgensen, H. S., Raaschou, H. O., and Olsen, T. S. (1994). Compensation in recovery of upper extremity function after stroke: the Copenhagen stroke study. Arch. Phys. Med. Rehabil. 75, 852-857. doi:10.1016/0003-9993(94) 90108-2

Nasseri, P., Nitsche, M. A., and Ekhtiari, H. (2015). A framework for categorizing electrode montages in transcranial direct current stimulation. Front. Hum. Neurosci. 9:54. doi:10.3389/fnhum.2015.00054

Nitsche, M. A., Cohen, L. G., Wassermann, E. M., Priori, A., Lang, N., Antal, A., et al. (2008). Transcranial direct current stimulation: state of the art 2008. Brain Stimulat. 1, 206-223. doi:10.1016/j.brs.2008.06.004

Nitsche, M. A., Schauenburg, A., Lang, N., Liebetanz, D., Exner, C., Paulus, W., et al. (2003). Facilitation of implicit motor learning by weak transcranial direct current stimulation of the primary motor cortex in the human. J. Cogn. Neurosci. 15, 619-626. doi:10.1162/089892903321662994

Nowak, D. A., Grefkes, C., Ameli, M., and Fink, G. R. (2009). Interhemispheric competition after stroke: brain stimulation to enhance recovery of function of the affected hand. Neurorehabil. Neural Repair 23, 641-656. doi:10.1177/ 1545968309336661

Nowak, D. A., Grefkes, C., Dafotakis, M., Eickhoff, S., Kust, J., Karbe, H., et al. (2008). Effects of low-frequency repetitive transcranial magnetic stimulation of the contralesional primary motor cortex on movement kinematics and neural activity in subcortical stroke. Arch. Neurol. 65, 741-747. doi:10.1001/archneur. 65.6.741

Nudo, R. J. (2006). Plasticity. NeuroRx 3, 420-427. doi:10.1016/j.nurx.2006.07.006

Nudo, R. J., and Milliken, G. W. (1996). Reorganization of movement representations in primary motor cortex following focal ischemic infarcts in adult squirrel monkeys. J. Neurophysiol. 75, 2144-2149.

Nudo, R. J., Wise, B. M., SiFuentes, F., and Milliken, G. W. (1996). Neural substrates for the effects of rehabilitative training on motor recovery after ischemic infarct. Science 272, 1791-1794. doi:10.1126/science.272.5269.1791

O'Doherty, J. P. (2004). Reward representations and reward-related learning in the human brain: insights from neuroimaging. Curr. Opin. Neurobiol. 14, 769-776. doi:10.1016/j.conb.2004.10.016

O’Shea, J., Boudrias, M. H., Stagg, C. J., Bachtiar, V., Kischka, U., Blicher, J. U., et al. (2014). Predicting behavioural response to TDCS in chronic motor stroke. Neuroimage 85(Pt 3), 924-933. doi:10.1016/j.neuroimage.2013.05.096

Pascual-Leone, A., Nguyet, D., Cohen, L. G., Brasil-Neto, J. P., Cammarota, A., and Hallett, M. (1995). Modulation of muscle responses evoked by transcranial magnetic stimulation during the acquisition of new fine motor skills. J. Neurophysiol. 74, 1037-1045.

Plow, E. B., Cunningham, D. A., Varnerin, N., and Machado, A. (2014). Rethinking stimulation of the brain in stroke rehabilitation: why higher motor areas might be better alternatives for patients with greater impairments. Neuroscientist doi: $10.1177 / 1073858414537381$

Reis, J., Schambra, H. M., Cohen, L. G., Buch, E. R., Fritsch, B., Zarahn, E., et al. (2009a). Noninvasive cortical stimulation enhances motor skill acquisition over multiple days through an effect on consolidation. Proc. Natl. Acad. Sci. U.S.A. 106, 1590-1595. doi:10.1073/pnas.0805413106

Reis, J., Schambra, H. M., Cohen, L. G., Buch, E. R., Fritsch, B., Zarahn, E., et al. (2009b). Noninvasive cortical stimulation enhances motor skill acquisition over multiple days through an effect on consolidation. Proc. Natl. Acad. Sci U.S.A. 106, 1590-1595. doi:10.1073/pnas.0805413106

Ridding, M. C., and Ziemann, U. (2010). Determinants of the induction of cortical plasticity by non-invasive brain stimulation in healthy subjects. J. Physiol. 588(Pt 13), 2291-2304. doi:10.1113/jphysiol.2010.190314

Rioult-Pedotti, M. S., Friedman, D., and Donoghue, J. P. (2000). Learning-induced LTP in neocortex. Science 290, 533-536. doi:10.1126/science.290.5491.533

Robertson, E. M., Pascual-Leone, A., and Miall, R. C. (2004a). Current concepts in procedural consolidation. Nat. Rev. Neurosci. 5, 576-582. doi:10. $1038 /$ nrn1426

Robertson, E. M., Pascual-Leone, A., and Press, D. Z. (2004b). Awareness modifies the skill-learning benefits of sleep. Curr. Biol. 14, 208-212. doi:10.1016/ S0960-9822(04)00039-9

Robertson, E. M., Press, D. Z., and Pascual-Leone, A. (2005). Off-line learning and the primary motor cortex. J. Neurosci. 25, 6372-6378. doi:10.1523/JNEUROSCI. 1851-05.2005

Romano, J. C., Howard, J. H. Jr., and Howard, D. V. (2010). One-year retention of general and sequence-specific skills in a probabilistic, serial reaction time task. Memory 18, 427-441. doi:10.1080/09658211003742680

Rose, D. K., Patten, C., McGuirk, T. E., Lu, X., and Triggs, W. J. (2014). Does inhibitory repetitive transcranial magnetic stimulation augment functional task practice to improve arm recovery in chronic stroke? Stroke Res. Treat. 2014, 305236. doi:10.1155/2014/305236

Rossi, C., Sallustio, F., Di Legge, S., Stanzione, P., and Koch, G. (2013). Transcranial direct current stimulation of the affected hemisphere does not accelerate recovery of acute stroke patients. Eur. J. Neurol. 20, 202-204. doi:10.1111/j.1468-1331. 2012.03703.x

Rossi, S., Hallett, M., Rossini, P. M., and Pascual-Leone, A. (2009). Safety of TMSCG. Safety, ethical considerations, and application guidelines for the use of transcranial magnetic stimulation in clinical practice and research. Neurophysiol. Clin. 120, 2008-2039. doi:10.1016/j.clinph.2009.08.016 
Ruffini, G., Fox, M. D., Ripolles, O., Miranda, P. C., and Pascual-Leone, A. (2014). Optimization of multifocal transcranial current stimulation for weighted cortical pattern targeting from realistic modeling of electric fields. Neuroimage 89, 216-225. doi:10.1016/j.neuroimage.2013.12.002

Sasaki, N., Mizutani, S., Kakuda, W., and Abo, M. (2013). Comparison of the effects of high- and low-frequency repetitive transcranial magnetic stimulation on upper limb hemiparesis in the early phase of stroke. J. Stroke Cerebrovasc. Dis. 22, 413-418. doi:10.1016/j.jstrokecerebrovasdis.2011.10.004

Schulz, R., Gerloff, C., and Hummel, F. C. (2013). Non-invasive brain stimulation in neurological diseases. Neuropharmacology 64, 579-587. doi:10.1016/j. neuropharm.2012.05.016

Schweighofer, N., Lee, J. Y., Goh, H. T., Choi, Y., Kim, S. S., Stewart, J. C., et al. (2011). Mechanisms of the contextual interference effect in individuals poststroke. J. Neurophysiol. 106, 2632-2641. doi:10.1152/jn.00399.2011

Seniow, J., Bilik, M., Lesniak, M., Waldowski, K., Iwanski, S., and Czlonkowska, A. (2012). Transcranial magnetic stimulation combined with physiotherapy in rehabilitation of poststroke hemiparesis: a randomized, double-blind, placebocontrolled study. Neurorehabil. Neural Repair 26, 1072-1079. doi:10.1177/ 1545968312445635

Shimizu, T., Hosaki, A., Hino, T., Sato, M., Komori, T., Hirai, S., et al. (2002). Motor cortical disinhibition in the unaffected hemisphere after unilateral cortical stroke. Brain 125(Pt 8), 1896-1907. doi:10.1093/brain/awf183

Sohn, M. K., Jee, S. J., and Kim, Y. W. (2013). Effect of transcranial direct current stimulation on postural stability and lower extremity strength in hemiplegic stroke patients. Ann. Rehabil. Med. 37, 759-765. doi:10.5535/arm.2013.37.6.759

Stagg, C. J., Bachtiar, V., O'Shea, J., Allman, C., Bosnell, R. A., Kischka, U., et al. (2012). Cortical activation changes underlying stimulation-induced behavioural gains in chronic stroke. Brain 135(Pt 1), 276-284. doi:10.1093/brain/awr313

Stagg, C. J., Jayaram, G., Pastor, D., Kincses, Z. T., Matthews, P. M., and JohansenBerg, H. (2011). Polarity and timing-dependent effects of transcranial direct current stimulation in explicit motor learning. Neuropsychologia 49, 800-804. doi:10.1016/j.neuropsychologia.2011.02.009

Stefan, K., Kunesch, E., Cohen, L. G., Benecke, R., and Classen, J. (2000). Induction of plasticity in the human motor cortex by paired associative stimulation. Brain 123(Pt 3), 572-584. doi:10.1093/brain/123.3.572

Stinear, C. M., Barber, P. A., Petoe, M., Anwar, S., and Byblow, W. D. (2012). The PREP algorithm predicts potential for upper limb recovery after stroke. Brain 135(Pt 8), 2527-2535. doi:10.1093/brain/aws146

Sung, W. H., Wang, C. P., Chou, C. L., Chen, Y. C., Chang, Y. C., and Tsai, P. Y. (2013). Efficacy of coupling inhibitory and facilitatory repetitive transcranial magnetic stimulation to enhance motor recovery in hemiplegic stroke patients. Stroke 44, 1375-1382. doi:10.1161/STROKEAHA.111.000522

Tahtis, V., Kaski, D., and Seemungal, B. M. (2014). The effect of single session bi-cephalic transcranial direct current stimulation on gait performance in subacute stroke: a pilot study. Restor. Neurol. Neurosci. 32, 527-532. doi:10.3233/ RNN-140393

Takeuchi, N., Chuma, T., Matsuo, Y., Watanabe, I., and Ikoma, K. (2005). Repetitive transcranial magnetic stimulation of contralesional primary motor cortex improves hand function after stroke. Stroke 36, 2681-2686. doi:10.1161/01.STR. 0000189658.51972 .34

Takeuchi, N., Tada, T., Toshima, M., Chuma, T., Matsuo, Y., and Ikoma, K. (2008). Inhibition of the unaffected motor cortex by $1 \mathrm{~Hz}$ repetitive transcranical magnetic stimulation enhances motor performance and training effect of the paretic hand in patients with chronic stroke. J. Rehabil. Med. 40, 298-303. doi:10.2340/16501977-0181

Takeuchi, N., Tada, T., Toshima, M., Matsuo, Y., and Ikoma, K. (2009). Repetitive transcranial magnetic stimulation over bilateral hemispheres enhances motor function and training effect of paretic hand in patients after stroke. J. Rehabil. Med. 41, 1049-1054. doi:10.2340/16501977-0454

Talelli, P., Greenwood, R. J., and Rothwell, J. C. (2007). Exploring theta burst stimulation as an intervention to improve motor recovery in chronic stroke. Neurophysiol. Clin. 118, 333-342. doi:10.1016/j.clinph.2006.10.014

Talelli, P., Wallace, A., Dileone, M., Hoad, D., Cheeran, B., Oliver, R., et al. (2012). Theta burst stimulation in the rehabilitation of the upper limb: a semirandomized, placebo-controlled trial in chronic stroke patients. Neurorehabil. Neural Repair 26, 976-987. doi:10.1177/1545968312437940

Tanaka, S., Takeda, K., Otaka, Y., Kita, K., Osu, R., Honda, M., et al. (2011). Single session of transcranial direct current stimulation transiently increases knee extensor force in patients with hemiparetic stroke. Neurorehabil. Neural Repair 25, 565-569. doi:10.1177/1545968311402091

Tecchio, F., Zappasodi, F., Assenza, G., Tombini, M., Vollaro, S., Barbati, G., et al. (2010). Anodal transcranial direct current stimulation enhances procedural consolidation. J. Neurophysiol. 104, 1134-1140. doi:10.1152/jn.00661.2009

Tempel, B. L., Bonini, N., Dawson, D. R., and Quinn, W. G. (1983). Reward learning in normal and mutant Drosophila. Proc. Natl. Acad. Sci. U.S.A. 80, 1482-1486. doi:10.1073/pnas.80.5.1482

Terney, D., Chaieb, L., Moliadze, V., Antal, A., and Paulus, W. (2008). Increasing human brain excitability by transcranial high-frequency random noise stimulation. J. Neurosci. 28, 14147-14155. doi:10.1523/JNEUROSCI.4248-08. 2008

Thomalla, G., Cheng, B., Ebinger, M., Hao, Q., Tourdias, T., Wu, O., et al. (2011). DWI-FLAIR mismatch for the identification of patients with acute ischaemic stroke within $4.5 \mathrm{~h}$ of symptom onset (PRE-FLAIR): a multicentre observational study. Lancet Neurol. 10, 978-986. doi:10.1016/S1474-4422(11)70192-2

Toni, N., Buchs, P. A., Nikonenko, I., Bron, C. R., and Muller, D. (1999). LTP promotes formation of multiple spine synapses between a single axon terminal and a dendrite. Nature 402, 421-425. doi:10.1038/46574

Ungerleider, L. G., Doyon, J., and Karni, A. (2002). Imaging brain plasticity during motor skill learning. Neurobiol. Learn. Mem. 78, 553-564. doi:10.1006/nlme. 2002.4091

Viana, R. T., Laurentino, G. E., Souza, R. J., Fonseca, J. B., Silva Filho, E. M., Dias, S. N., et al. (2014). Effects of the addition of transcranial direct current stimulation to virtual reality therapy after stroke: a pilot randomized controlled trial. NeuroRehabilitation 34, 437-446. doi:10.3233/NRE-141065

Vollmann, H., Conde, V., Sewerin, S., Taubert, M., Sehm, B., Witte, O. W., et al. (2013). Anodal transcranial direct current stimulation (tDCS) over supplementary motor area (SMA) but not pre-SMA promotes short-term visuomotor learning. Brain Stimulat. 6, 101-107. doi:10.1016/j.brs.2012.03.018

Wagner, T., Fregni, F., Fecteau, S., Grodzinsky, A., Zahn, M., and Pascual-Leone, A. (2007). Transcranial direct current stimulation: a computer-based human model study. Neuroimage 35, 1113-1124. doi:10.1016/j.neuroimage.2007.01.027

Wang, C. C., Wang, C. P., Tsai, P. Y., Hsieh, C. Y., Chan, R. C., and Yeh, S. C. (2014). Inhibitory repetitive transcranial magnetic stimulation of the contralesional premotor and primary motor cortices facilitate poststroke motor recovery. Restor Neurol. Neurosci. 32, 825-835. doi:10.3233/RNN-140410

Wang, R. Y., Tseng, H. Y., Liao, K. K., Wang, C. J., Lai, K. L., and Yang, Y. R. (2012). rTMS combined with task-oriented training to improve symmetry of interhemispheric corticomotor excitability and gait performance after stroke: a randomized trial. Neurorehabil. Neural Repair 26, 222-230. doi:10.1177/ 1545968311423265

Ward, N. S. (2004). Functional reorganization of the cerebral motor system after stroke. Curr. Opin. Neurol. 17, 725-730. doi:10.1097/ 00019052-200412000-00013

Ward, N. S., Brown, M. M., Thompson, A. J., and Frackowiak, R. S. (2003). Neural correlates of motor recovery after stroke: a longitudinal fMRI study. Brain $126(\mathrm{Pt}$ 11), 2476-2496. doi:10.1093/brain/awg145

Ward, N. S., Brown, M. M., Thompson, A. J., and Frackowiak, R. S. (2004). The influence of time after stroke on brain activations during a motor task. Ann. Neurol. 55, 829-834. doi:10.1002/ana.20099

Wassermann, E. M., Grafman, J., Berry, C., Hollnagel, C., Wild, K., Clark, K., et al. (1996). Use and safety of a new repetitive transcranial magnetic stimulator. Electroencephalogr. Clin. Neurophysiol. 101, 412-417. doi:10.1016/0924-980X(96) 96004-X

Wessel, M., Zimerman, M., Timmermann, J. E., and Hummel, F. C. (2013). Eyelid myokymia in an older subject after repetitive sessions of anodal transcranial direct current stimulation. Brain Stimulat. 6, 463-465. doi:10.1016/j.brs.2012. 09.002

Wessel, M. J., Zimerman, M., Timmermann, J. E., Heise, K. F., Gerloff, C., and Hummel, F. C. (2015). Enhancing consolidation of a new temporal motor skill by cerebellar noninvasive stimulation. Cereb. Cortex pii, bhu335. doi:10.1093/ cercor/bhu335

Wiethoff, S., Hamada, M., and Rothwell, J. C. (2014). Variability in response to transcranial direct current stimulation of the motor cortex. Brain Stimulat. 7, 468-475. doi:10.1016/j.brs.2014.02.003

Willingham, D. B. (1998). A neuropsychological theory of motor skill learning. Psychol. Rev. 105, 558-584. doi:10.1037/0033-295X.105.3.558 
Wymbs, N. F., and Grafton, S. T. (2009). Neural substrates of practice structure that support future off-line learning. J. Neurophysiol. 102, 2462-2476. doi:10.1152/ jn.00315.2009

Yozbatiran, N., Alonso-Alonso, M., See, J., Demirtas-Tatlidede, A., Luu, D., Motiwala, R. R., et al. (2009). Safety and behavioral effects of high-frequency repetitive transcranial magnetic stimulation in stroke. Stroke 40, 309-312. doi:10. 1161/STROKEAHA.108.522144

Zald, D. H., Boileau, I., El-Dearedy, W., Gunn, R., McGlone, F., Dichter, G. S., et al. (2004). Dopamine transmission in the human striatum during monetary reward tasks. J. Neurosci. 24, 4105-4112. doi:10.1523/JNEUROSCI.4643-03. 2004

Ziemann, U., Ilic, T. V., Pauli, C., Meintzschel, F., and Ruge, D. (2004). Learning modifies subsequent induction of long-term potentiation-like and long-term depression-like plasticity in human motor cortex. J. Neurosci. 24, 1666-1672. doi:10.1523/JNEUROSCI.5016-03.2004

Ziemann, U., Paulus, W., Nitsche, M. A., Pascual-Leone, A., Byblow, W. D., Berardelli, A., et al. (2008). Consensus: motor cortex plasticity protocols. Brain Stimulat. 1, 164-182. doi:10.1016/j.brs.2008.06.006
Zimerman, M., Heise, K. F., Hoppe, J., Cohen, L. G., Gerloff, C., and Hummel, F. C. (2012). Modulation of training by single-session transcranial direct current stimulation to the intact motor cortex enhances motor skill acquisition of the paretic hand. Stroke 43, 2185-2191. doi:10.1161/STROKEAHA.111.645382

Zimerman, M., Nitsch, M., Giraux, P., Gerloff, C., Cohen, L. G., and Hummel, F. C. (2013). Neuroenhancement of the aging brain: restoring skill acquisition in old subjects. Ann. Neurol. 73, 10-15. doi:10.1002/ana.23761

Conflict of Interest Statement: The authors declare that the research was conducted in the absence of any commercial or financial relationships that could be construed as a potential conflict of interest.

Copyright (c) 2015 Wessel, Zimerman and Hummel. This is an open-access article distributed under the terms of the Creative Commons Attribution License (CC BY). The use, distribution or reproduction in other forums is permitted, provided the original author(s) or licensor are credited and that the original publication in this journal is cited, in accordance with accepted academic practice. No use, distribution or reproduction is permitted which does not comply with these terms. 